\title{
REVISED RNA-seq analysis is easy as 1-2-3 with limma, Glimma
}

\section{and edgeR [version 2; peer review: 3 approved]}

\section{Charity W. Law (D1,2, Monther Alhamdoosh (Di)3, Shian Su(D1), Gordon K. Smyth (D1,4, Matthew E. Ritchie (D) 1,2,4}

\author{
${ }^{1}$ The Walter and Eliza Hall Institute of Medical Research, Parkville, 3052, Australia \\ 2Department of Medical Biology, The University of Melbourne, Parkville, 3010, Australia \\ ${ }^{3}$ CSL Limited, Parkville, Victoria, 3010, Australia \\ ${ }^{4}$ School of Mathematics and Statistics, The University of Melbourne, Parkville, 3010, Australia
}

V2 First published: 17 Jun 2016, 5:1408

https://doi.org/10.12688/f1000research.9005.1

Second version: 30 Nov 2016, 5:1408

https://doi.org/10.12688/f1000research.9005.2

Latest published: 28 Dec 2018, 5:1408

https://doi.org/10.12688/f1000research.9005.3

\section{Abstract}

The ability to easily and efficiently analyse RNA-sequencing data is a key strength of the Bioconductor project. Starting with counts summarised at the gene-level, a typical analysis involves preprocessing, exploratory data analysis, differential expression testing and pathway analysis with the results obtained informing future experiments and validation studies. In this workflow article, we analyse RNA-sequencing data from the mouse mammary gland, demonstrating use of the popular edgeR package to import, organise, filter and normalise the data, followed by the limma package with its voom method, linear modelling and empirical Bayes moderation to assess differential expression and perform gene set testing. This pipeline is further enhanced by the Glimma package which enables interactive exploration of the results so that individual samples and genes can be examined by the user. The complete analysis offered by these three packages highlights the ease with which researchers can turn the raw counts from an RNA-sequencing experiment into biological insights using Bioconductor.

\section{Keywords}

RNA sequencing, data analysis, gene expression

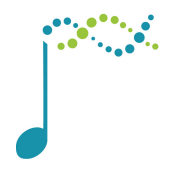

This article is included in the Bioconductor

gateway.

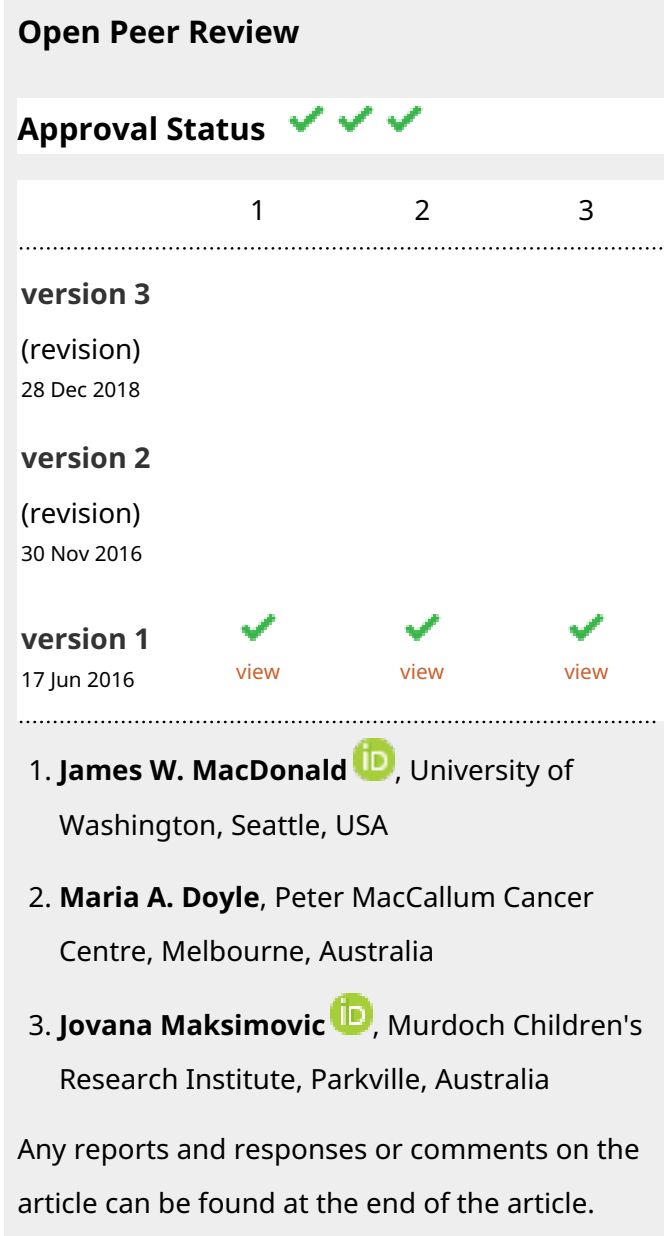


gateway.

Corresponding authors: Gordon K. Smyth (smyth@wehi.edu.au), Matthew E. Ritchie (mritchie@wehi.edu.au)

Competing interests: The authors declare that they have no competing interests.

Grant information: This work was funded by the National Health and Medical Research Council (NHMRC) (Fellowship GNT1058892 and Program GNT1054618 to GKS, Project GNT1050661 to MER and GKS and Fellowship GNT1104924 to MER), Victorian State Government Operational Infrastructure Support and Australian Government NHMRC IRIISS.

The funders had no role in study design, data collection and analysis, decision to publish, or preparation of the manuscript.

Copyright: $\odot 2016$ Law CW et al. This is an open access article distributed under the terms of the Creative Commons Attribution License, which permits unrestricted use, distribution, and reproduction in any medium, provided the original work is properly cited.

How to cite this article: Law CW, Alhamdoosh M, Su S et al. RNA-seq analysis is easy as 1-2-3 with limma, Glimma and edgeR [version 2; peer review: 3 approved] F1000Research 2016, 5:1408 https://doi.org/10.12688/f1000research.9005.2

First published: 17 Jun 2016, 5:1408 https://doi.org/10.12688/f1000research.9005.1 


\section{REVISED Amendments from Version 1}

The article has been updated with minor edits to the R code for simplification and minor text changes to clarify any ambiguous wording. Extra information has been added to the section on "Gene set testing with camera" to briefly describe c2 and hallmark gene sets and to explain how to choose between camera and mroast, another gene set testing method available in limma. In Figure 6, the table in Glimma's mean-difference plot has been updated to reflect the simplification of R code upstream in the "Organising gene annotations" section. Author contributions have also been specified in the update.

See referee reports

\section{Introduction}

RNA-sequencing (RNA-seq) has become the primary technology used for gene expression profiling, with the genomewide detection of differentially expressed genes between two or more conditions of interest one of the most commonly asked questions by researchers. The edge $\mathbf{R}^{1}$ and limma packages ${ }^{2}$ available from the Bioconductor project ${ }^{3}$ offer a well-developed suite of statistical methods for dealing with this question for RNA-seq data. In this article, we describe an edgeR - limma workflow for analysing RNA-seq data that takes gene-level counts as its input, and moves through pre-processing and exploratory data analysis before obtaining lists of differentially expressed (DE) genes and gene signatures. This analysis is enhanced through the use of interactive graphics from the Glimma package ${ }^{4}$, that allows for a more detailed exploration of the data at both the sample and gene-level than is possible using static R plots.

The experiment analysed in this workflow is from Sheridan et al. $(2015)^{5}$ and consists of three cell populations (basal, luminal progenitor (LP) and mature luminal (ML)) sorted from the mammary glands of female virgin mice, each profiled in triplicate. RNA samples were sequenced across three batches on an Illumina HiSeq 2000 to obtain 100 base-pair single-end reads. The analysis outlined in this article assumes that reads obtained from an RNA-seq experiment have been aligned to an appropriate reference genome and summarised into counts associated with gene-specific regions. In this instance, reads were aligned to the mouse reference genome $(\mathrm{mm} 10)$ using the $\mathrm{R}$ based pipeline available in the Rsubread package (specifically the align function ${ }^{6}$ followed by featureCount $s^{7}$ for gene-level summarisation based on the in-built $\mathrm{mm} 10$ RefSeq-based annotation). Count data for these samples can be downloaded from the Gene Expression Omnibus (GEO) using GEO Series accession number GSE63310. Further information on experimental design and sample preparation is also available from GEO under this accession number.

\section{Data packaging}

\section{Reading in count data}

To get started with this analysis, download the file GSE63310_RAW. tar available online from http://www.ncbi.nlm. nih.gov/geo/download/?acc=GSE63310\&format=file, and extract the relevant files from this archive. Each of these text files contains the raw gene-level counts for a given sample. Note that our analysis only includes the basal, LP and ML samples from this experiment (see associated file names below).

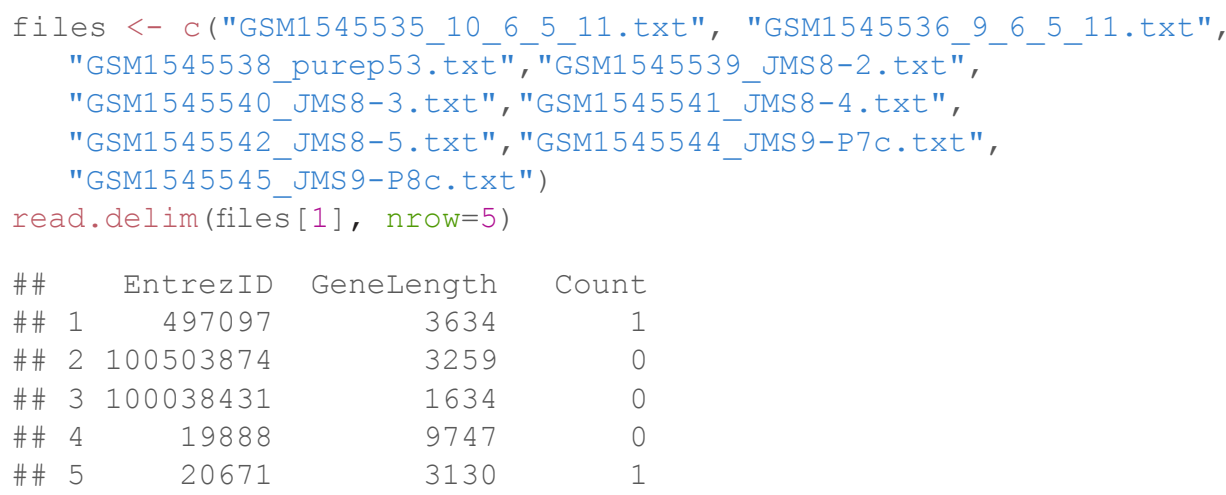

Whilst each of the nine text files can be read into R separately and combined into a matrix of counts, edgeR offers a convenient way to do this in one step using the readDGE function. The resulting DGEList-object contains a matrix of counts with 27,179 rows associated with unique Entrez gene identifiers (IDs) and nine columns associated with the individual samples in the experiment. 


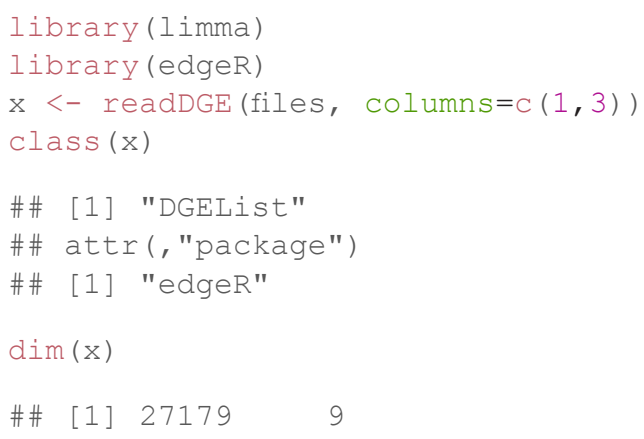

If the counts from all samples were stored in a single file, the data can be read into $\mathrm{R}$ and then converted into a DGEList-object using the DGEList function.

\section{Organising sample information}

For downstream analysis, sample-level information related to the experimental design needs to be associated with the columns of the counts matrix. This should include experimental variables, both biological and technical, that could have an effect on expression levels. Examples include cell type (basal, LP and ML in this experiment), genotype (wild-type, knock-out), phenotype (disease status, sex, age), sample treatment (drug, control) and batch information (date experiment was performed if samples were collected and analysed at distinct time points) to name just a few.

Our DGEList-object contains a samples data frame that stores both cell type (or group) and batch (sequencing lane) information, each of which consists of three distinct levels. Note that within $x \$ s a m p l e s$, library sizes are automatically calculated for each sample and normalisation factors are set to 1. For simplicity, we remove the GEO sample IDs (GSM*) from the column names of our DGEList-object $x$.

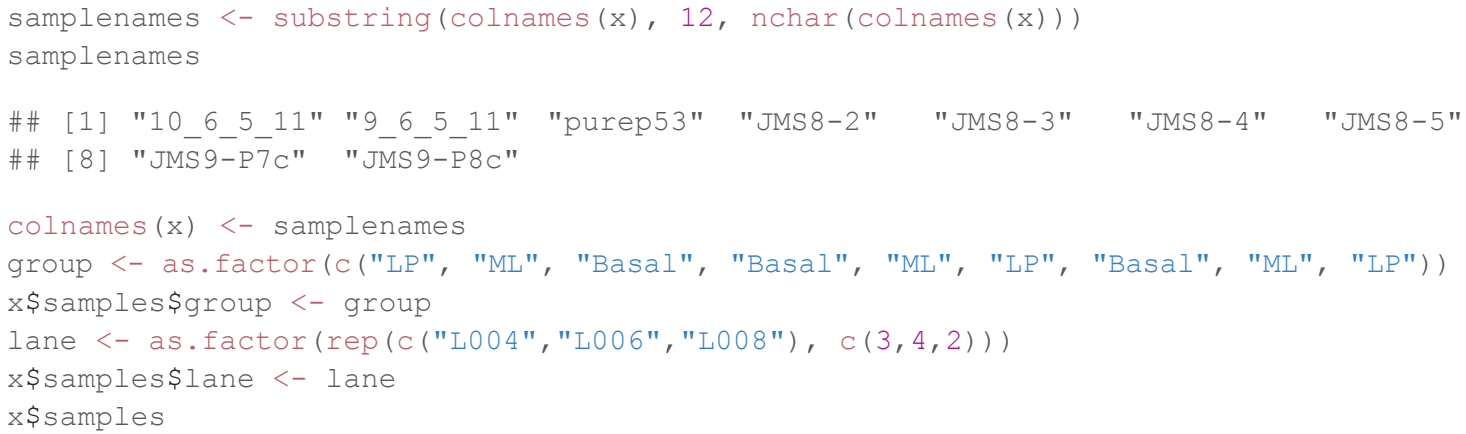

\begin{tabular}{|c|c|c|c|c|}
\hline files & group & lib.size & norm.factors & lane \\
\hline SM1545535 106511 .txt & LP & 32863052 & 1 & $\mathrm{~L} 004$ \\
\hline GSM1545536_9_6_5_11.txt & ML & 35335491 & 1 & L004 \\
\hline GSM1 $54553 \overline{8}$ purep 53. txt & Basal & 57160817 & 1 & L004 \\
\hline GSM1545539_JMS8-2.txt & Basal & 51368625 & 1 & L006 \\
\hline GSM1545540 JMS8-3.txt & ML & 75795034 & 1 & L006 \\
\hline GSM1545541_JMS8-4.txt & LP & 60517657 & 1 & L006 \\
\hline GSM1545542_JMS8-5.txt & Basal & & 1 & L006 \\
\hline GSM1545544_JMS9-P7C.txt & $\mathrm{ML}$ & 21311068 & 1 & L008 \\
\hline GSM1545545_JMS9-P8C.txt & LP & 8838 & 1 & L008 \\
\hline
\end{tabular}

Organising gene annotations

A second data frame named genes in the DGEList-object is used to store gene-level information associated with rows of the counts matrix. This information can be retrieved using organism specific packages such as Mus.musculus ${ }^{8}$ for mouse (or Homo.sapiens ${ }^{9}$ for human) or the biomaRt package ${ }^{10,11}$ which interfaces the Ensembl genome databases in order to perform gene annotation. The type of information that can be retrieved includes gene symbols, gene names, chromosome names and locations, Entrez gene IDs, Refseq gene IDs and Ensembl gene IDs to name just a few. biomaRt primarily works off Ensembl gene IDs, whereas Mus.musculus packages information from various sources and allows users to choose between many different gene IDs as the key. The Entrez gene IDs available in our dataset were annotated using the Mus.musculus package to retrieve associated gene symbols and chromosome information. 


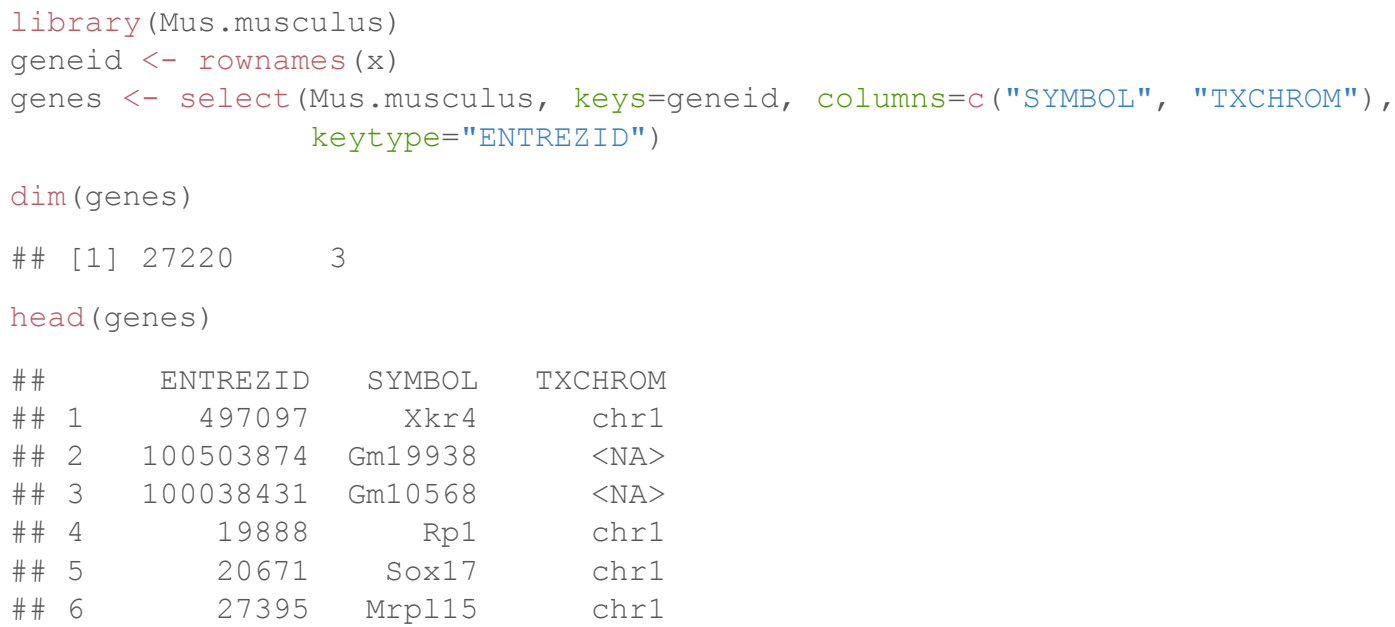

As with any gene ID, Entrez gene IDs may not map one-to-one to the gene information of interest. It is important to check for duplicated gene IDs and to understand the source of duplication before resolving them. Our gene annotation contains 28 genes that map to multiple chromosomes (e.g. gene Gm1987 is associated with "chr4" and “chr4_JH584294_random" and microRNA Mir5098 is associated with “chr2", "chr5", "chr8”, "chr11" and "chr17”). To resolve duplicate gene IDs one could combine all chromosome information from the multi-mapped genes, such that gene Gm1987 would be is assigned to "chr4 and chr4_JH584294_random", or select one of the chromosomes to represent the gene with duplicate annotation. For simplicity we do the latter, keeping only the first occurrence of each gene ID.

genes <- genes [ duplicated (genes\$ENTREZID), ]

In this example, the gene order is the same in both the annotation and the data object. If this is not the case due to missing and/or rearranged gene IDs, the match function can be used to order genes correctly. The data frame of gene annotations is then added to the data object and neatly packaged in a DGEList-object containing raw count data with associated sample information and gene annotations.

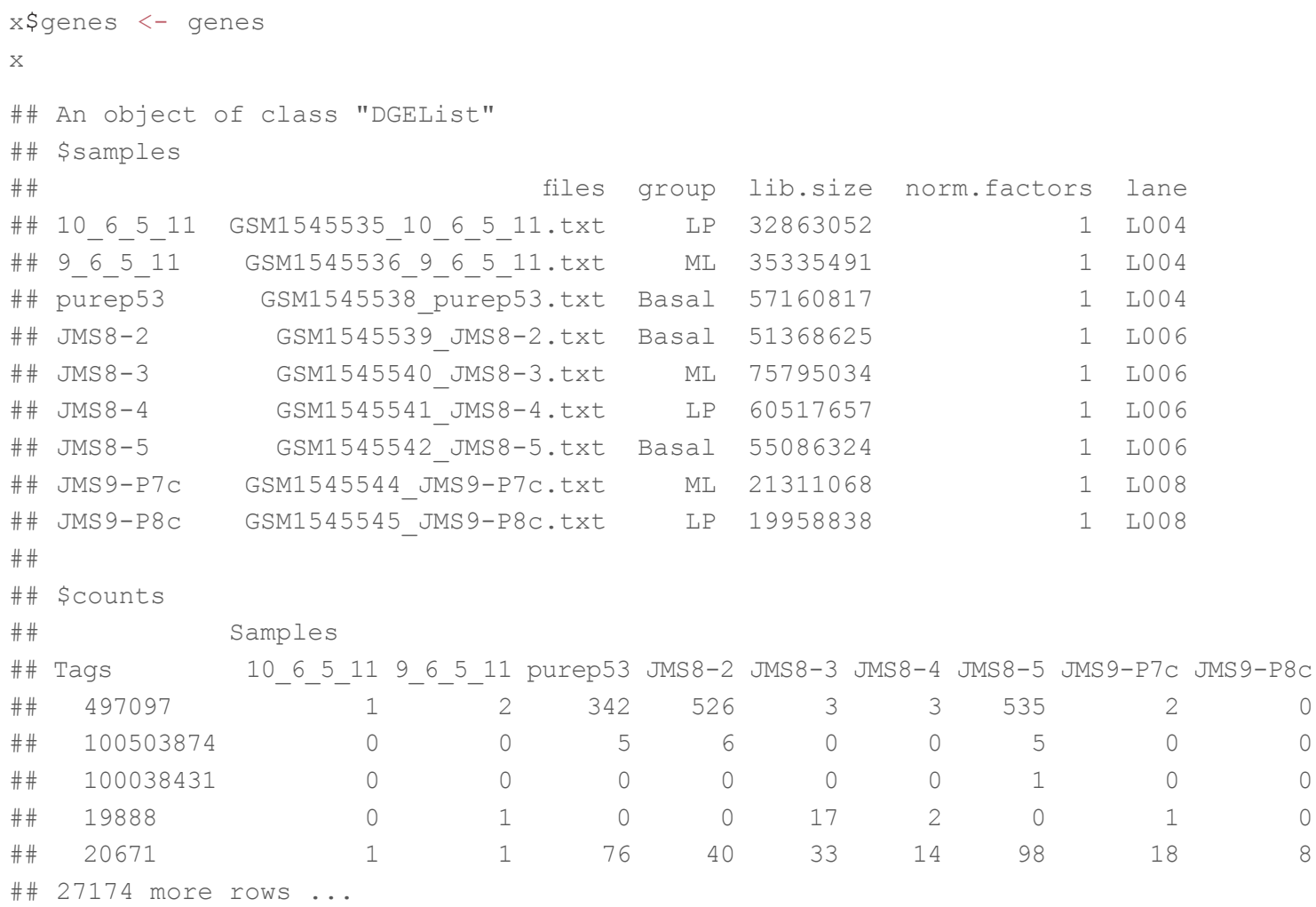




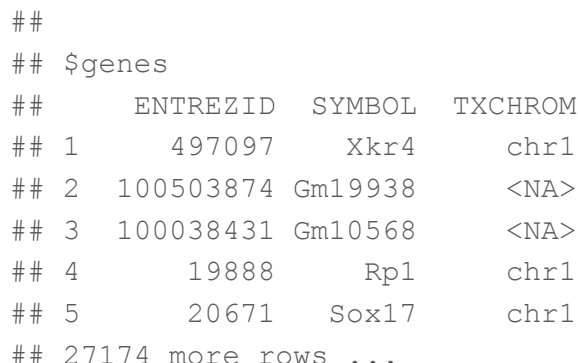

Data pre-processing

Transformations from the raw-scale

For differential expression and related analyses, gene expression is rarely considered at the level of raw counts since libraries sequenced at a greater depth will result in higher counts. Rather, it is common practice to transform raw counts onto a scale that accounts for such library size differences. Popular transformations include counts per million (CPM), $\log _{2}$-counts per million (log-CPM), reads per kilobase of transcript per million (RPKM), and fragments per kilobase of transcript per million (FPKM).

In our analyses, CPM and log-CPM transformations are used regularly although they do not account for feature length differences which RPKM and FPKM values do. Whilst RPKM and FPKM values can just as well be used, CPM and $\log$-CPM values can be calculated using a counts matrix alone and will suffice for the type of comparisons we are interested in. Assuming that there are no differences in isoform usage between conditions, differential expression analyses look at gene expression changes between conditions rather than comparing expression across multiple genes or drawing conclusions on absolute levels of expression. In other words, gene lengths remain constant for comparisons of interest and any observed differences are a result of changes in condition rather than changes in gene length.

Here raw counts are converted to CPM and $\log$-CPM values using the cpm function in edgeR, where $\log$ transformations use a prior count of 0.25 to avoid taking the log of zero. RPKM values are just as easily calculated as CPM values using the rp km function in edgeR if gene lengths are available.

cpm $<-\operatorname{cpm}(\mathrm{x})$

$1 \mathrm{cpm}<-\operatorname{cpm}(\mathrm{x}, \log =\mathrm{TRUE})$

Removing genes that are lowly expressed

All datasets will include a mix of genes that are expressed and those that are not expressed. Whilst it is of interest to examine genes that are expressed in one condition but not in another, some genes are unexpressed throughout all samples. In fact, $19 \%$ of genes in this dataset have zero counts across all nine samples.

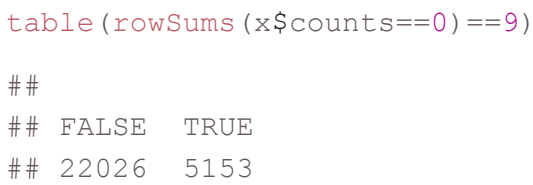

Genes that are not expressed at a biologically meaningful level in any condition should be discarded to reduce the subset of genes to those that are of interest, and to reduce the number of tests carried out downstream when looking at differential expression. Upon examination of log-CPM values, it can be seen that a large proportion of genes within each sample is unexpressed or lowly-expressed (Figure 1A). Genes are deemed to be expressed if their CPM value is above a threshold (we use a nominal CPM value of 1), and unexpressed otherwise. A CPM value of 1 is equivalent to a $\log$-CPM value of 0 . Genes must be expressed in at least one group (or in at least three samples across the entire experiment, where three was chosen as this is the smallest group size) to be kept for downstream analysis.

Although any sensible value can be used as the expression cutoff, typically a CPM value of 1 is used in our analyses as it separates expressed genes from unexpressed genes well for most datasets. Here, a CPM value of 1 means that a gene is "expressed" if it has at least 20 counts in the sample with the lowest sequencing depth (JMS9-P8c, library size $\approx 20$ million) or at least 76 counts in the sample with the greatest sequencing depth (JMS8-3, library size $\approx 76$ million). If sequence reads are summarised by exons rather than genes and/or experiments have low sequencing depth, a lower CPM cutoff may be considered. 
A. Raw data

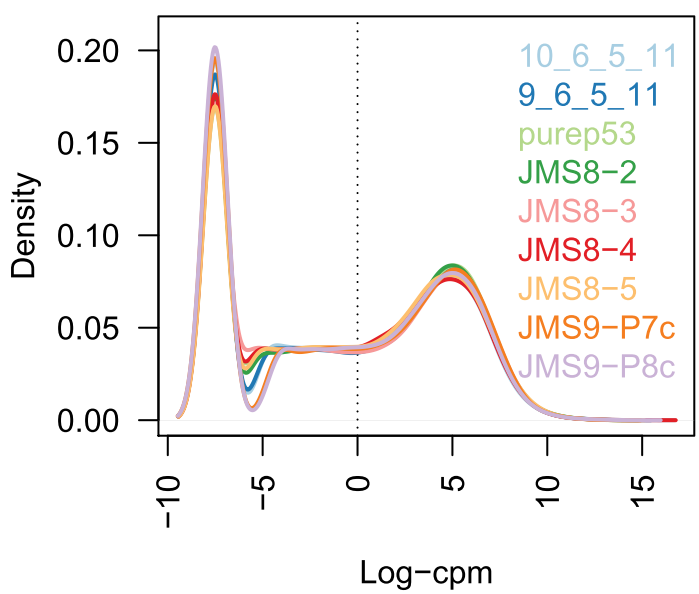

B. Filtered data

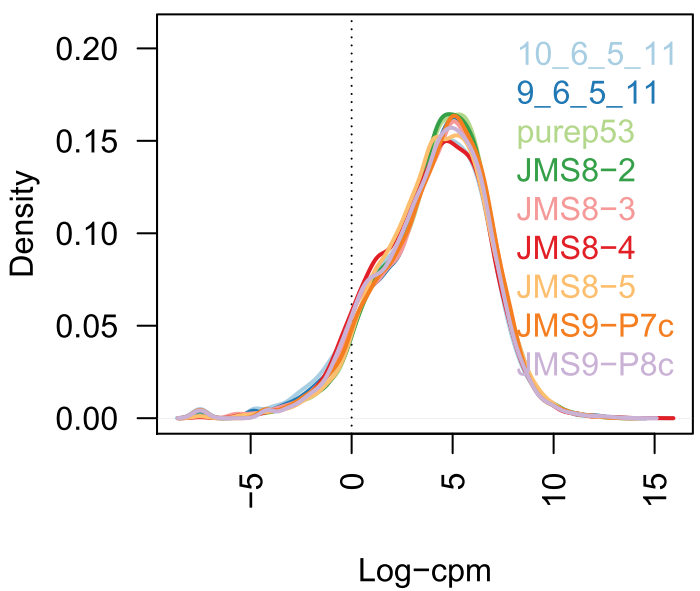

Figure 1. The density of log-CPM values for raw pre-filtered data (A) and post-filtered data (B) are shown for each sample. Dotted vertical lines mark the log-CPM of zero threshold (equivalent to a CPM value of 1) used in the filtering step.

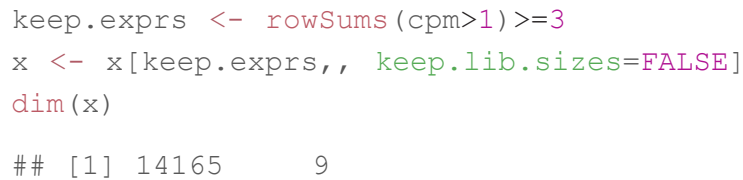

Using this criterion, the number of genes is reduced to approximately half the number that we started with $(14,165$ genes, Figure 1B). Note that subsetting the entire DGEList-object removes both the counts as well as the associated gene information. Code to produce Figure 1 is given below.

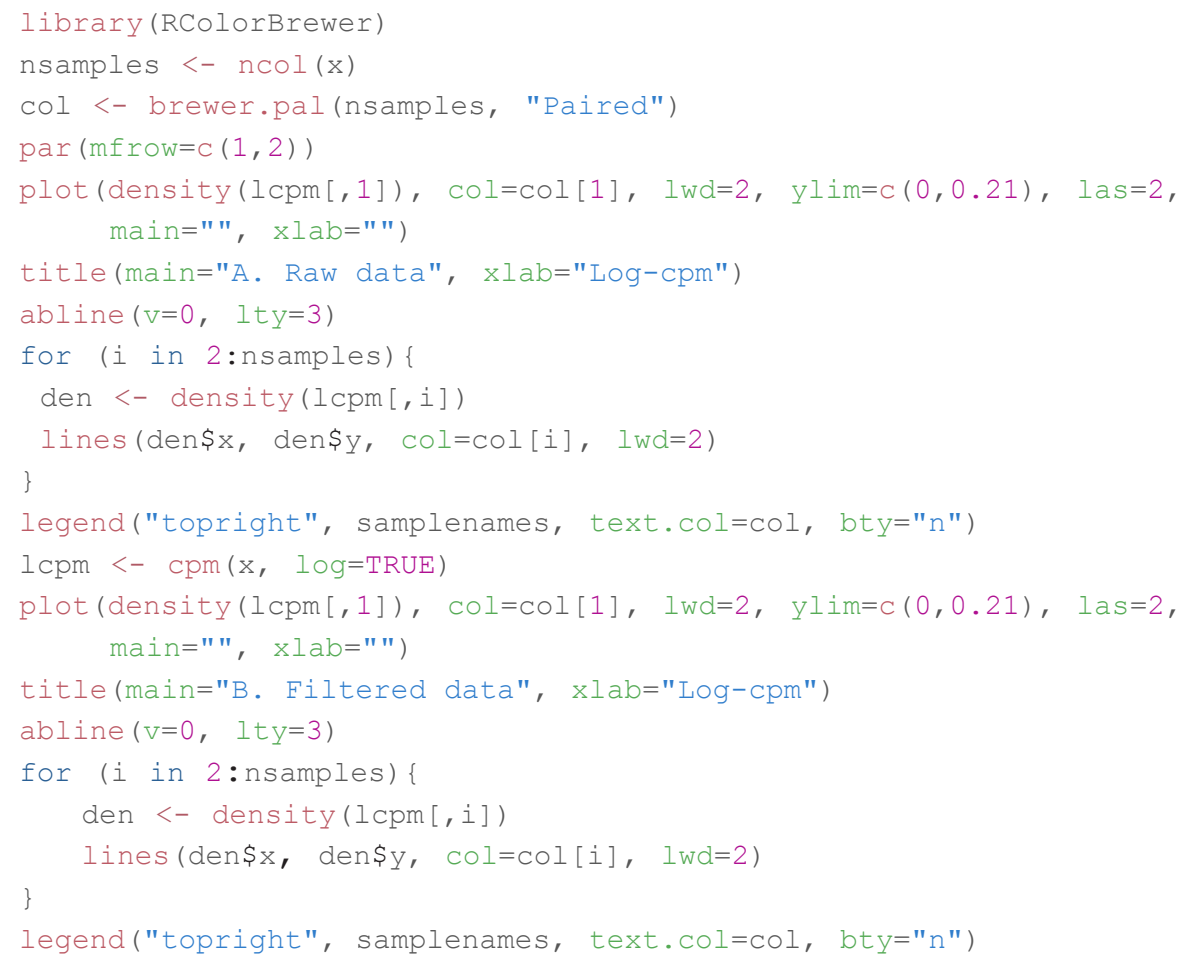


Normalising gene expression distributions

During the sample preparation or sequencing process, external factors that are not of biological interest can affect the expression of individual samples. For example, samples processed in the first batch of an experiment can have higher expression overall when compared to samples processed in a second batch. It is assumed that all samples should have a similar range and distribution of expression values. Normalisation is required to ensure that the expression distributions of each sample are similar across the entire experiment.

Any plot showing the per sample expression distributions, such as a density or boxplot, is useful in determining whether any samples are dissimilar to others. Distributions of log-CPM values are similar throughout all samples within this dataset (Figure 1B).

Nonetheless, normalisation by the method of trimmed mean of M-values (TMM) ${ }^{12}$ is performed using the calcNormFactors function in edgeR. The normalisation factors calculated here are used as a scaling factor for the library sizes. When working with DGEList-objects, these normalisation factors are automatically stored in $x$ \$samples $\$$ norm. factors. For this dataset the effect of TMM-normalisation is mild, as evident in the magnitude of the scaling factors, which are all relatively close to 1 .

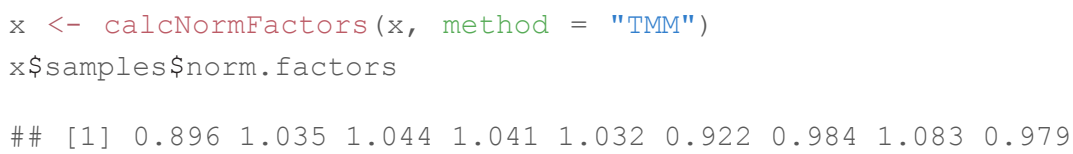

To give a better visual representation of the effects of normalisation, the data was duplicated then adjusted so that the counts of the first sample are reduced to $5 \%$ of their original values, and in the second sample they are inflated to be 5-times larger.

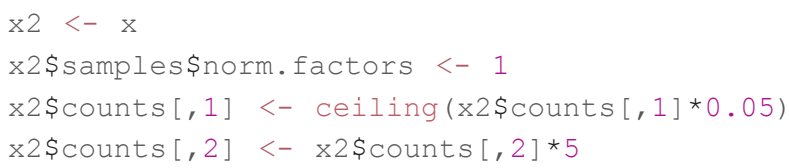

Figure 2 shows the expression distribution of samples for unnormalised and normalised data, where distributions are noticeably different pre-normalisation and are similar post-normalisation. Here the first sample has a small TMM scaling factor of 0.05 , whereas the second sample has a large scaling factor of 6.13 - neither values are close to 1 .

A. Example: Unnormalised data

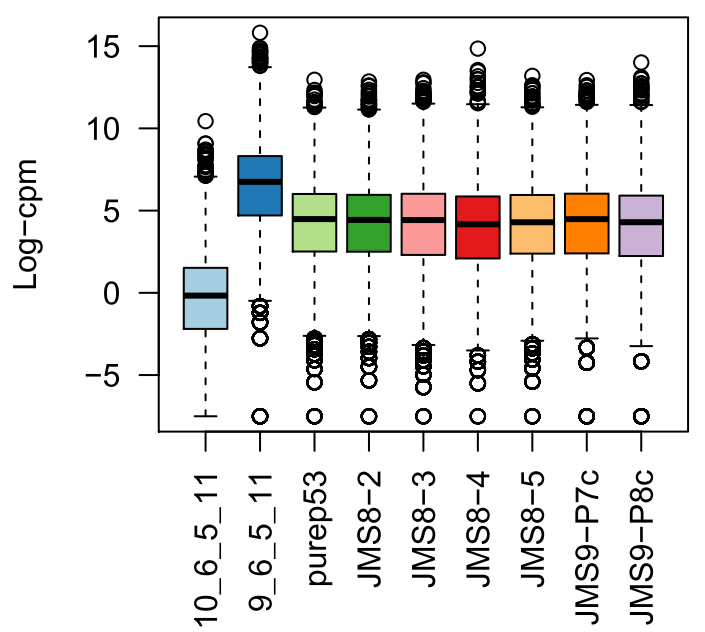

B. Example: Normalised data

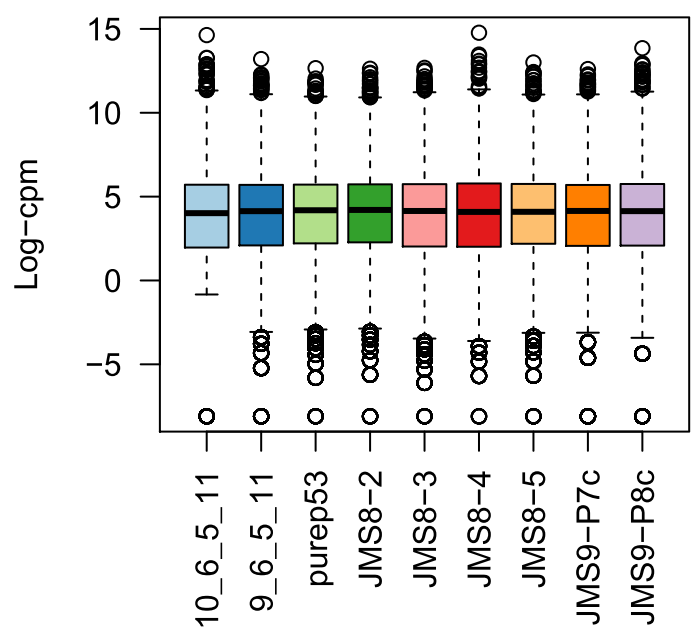

Figure 2. Example data: Boxplots of log-CPM values showing expression distributions for unnormalised data $(\mathbf{A})$ and normalised data (B) for each sample in the modified dataset where the counts in samples 1 and 2 have been scaled to $5 \%$ and $500 \%$ of their original values respectively. 


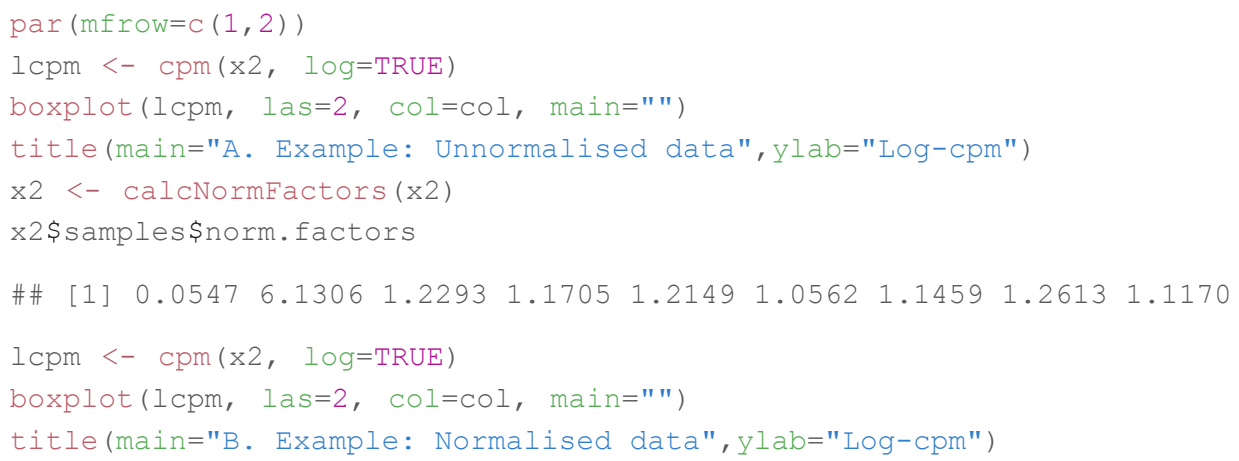

Unsupervised clustering of samples

In our opinion, one of the most important exploratory plots to examine for gene expression analyses is the multidimensional scaling (MDS) plot, or similar. The plot shows similarities and dissimilarities between samples in an unsupervised manner so that one can have an idea of the extent to which differential expression can be detected before carrying out formal tests. Ideally, samples would cluster well within the primary condition of interest, and any sample straying far from its group could be identified and followed up for sources of error or extra variation. If present, technical replicates should lie very close to one another.

Such a plot can be made in limma using the plotMDS function. The first dimension represents the leadingfold-change that best separates samples and explains the largest proportion of variation in the data, with subsequent dimensions having a smaller effect and being orthogonal to the ones before it. When experimental design involves multiple factors, it is recommended that each factor is examined over several dimensions. If samples cluster by a given factor in any of these dimensions, it suggests that the factor contributes to expression differences and is worth including in the linear modelling. On the other hand, factors that show little or no effect may be left out of downstream analysis.

In this dataset, samples can be seen to cluster well within experimental groups over dimension 1 and 2, and then separate by sequencing lane (sample batch) over dimension 3 (Figure 3). Keeping in mind that the first dimension explains the largest proportion of variation in the data, notice that the range of values over the dimensions become smaller as we move to higher dimensions. Whilst all samples cluster by groups, the largest transcriptional difference is observed between basal and LP, and basal and ML over dimension 1. For this reason, it is expected that pairwise comparisons between cell populations will result in a greater number of DE genes for comparisons involving basal

\section{A. Sample groups}

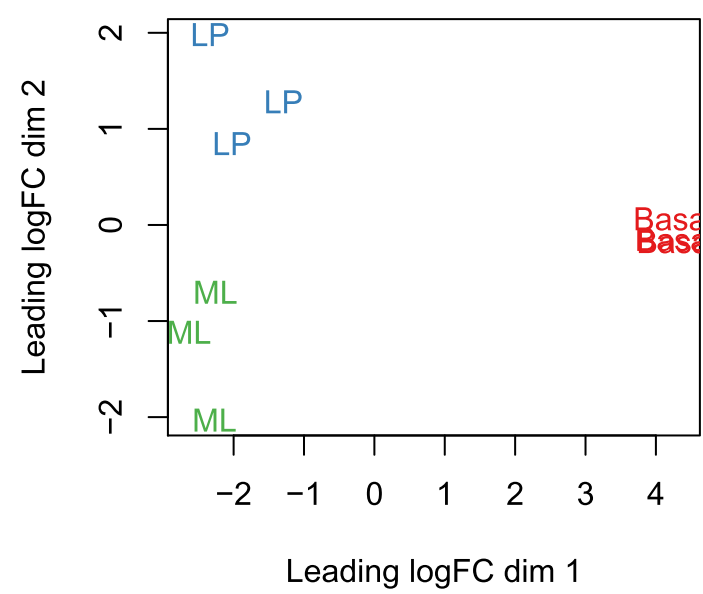

\section{B. Sequencing lanes}

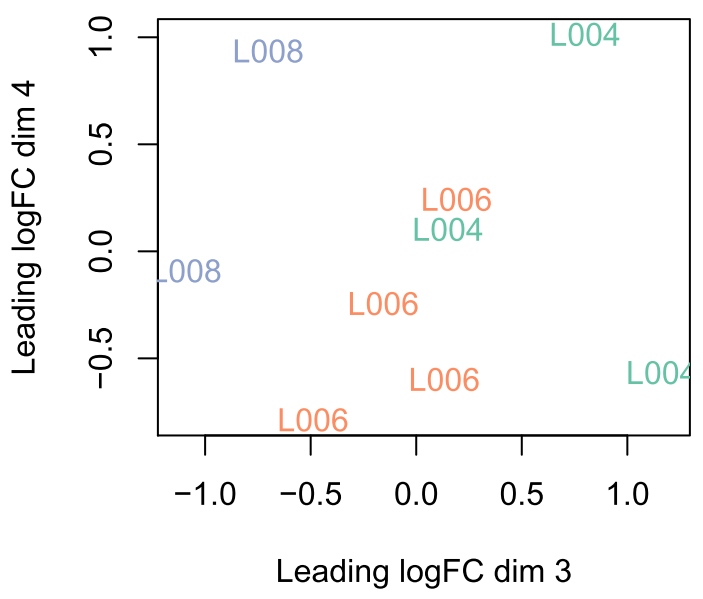

Figure 3. MDS plots of log-CPM values over dimensions 1 and 2 with samples coloured and labeled by sample groups (A) and over dimensions 3 and 4 with samples coloured and labeled by sequencing lane (B). Distances on the plot correspond to the leading fold-change, which is the average (root-mean-square) $\log _{2}$-fold-change for the 500 genes most divergent between each pair of samples by default. 
samples, and relatively small numbers of DE genes when comparing ML to LP. Datasets where samples do not cluster by experimental group may show little or no evidence of differential expression in the downstream analysis.

To create the MDS plots, we assign different colours to the factors of interest. Dimensions 1 and 2 are examined using the colour grouping defined by cell types.

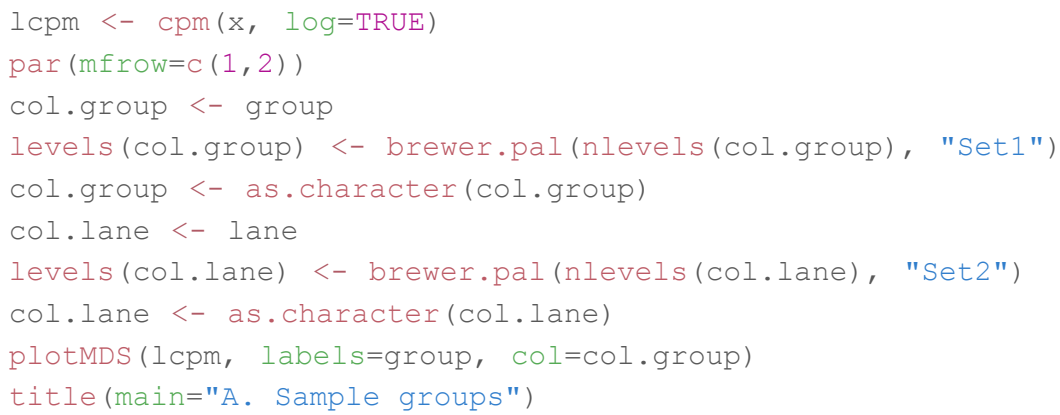

Dimensions 3 and 4 are examined using the colour grouping defined by sequencing lanes (batch).

plotMDS(lcpm, labels=lane, $\operatorname{col}=\operatorname{col}$. lane, $\operatorname{dim}=c(3,4))$

title (main="B. Sequencing lanes")

Alternatively, the Glimma package offers the convenience of an interactive MDS plot where multiple dimensions can be explored. The glMDSPlot function generates an html page (that is opened in a browser if launch=TRUE) with an MDS plot in the left panel and a barplot showing the proportion of variation explained by each dimension in the right panel. Clicking on the bars of the bar plot changes the pair of dimensions plotted in the MDS plot, and hovering over the individual points reveals the sample label. The colour scheme can be changed as well to highlight cell population or sequencing lane (batch). An interactive MDS plot of this dataset can be found at http://bioinf.wehi.edu.au/folders/limmaWorkflow/glimma-plots/MDS-Plot.html.

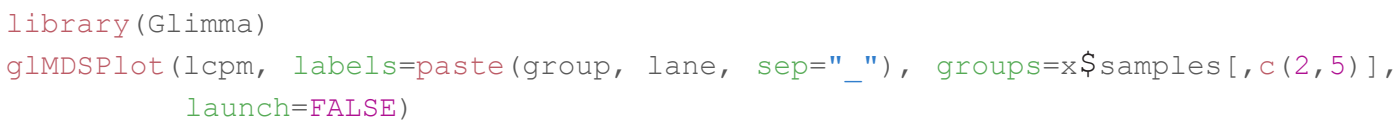

Differential expression analysis

Creating a design matrix and contrasts

In this study, it is of interest to see which genes are expressed at different levels between the three cell populations profiled. In our analysis, linear models are fitted to the data with the assumption that the underlying data is normally distributed. To get started, a design matrix is set up with both the cell population and sequencing lane (batch) information.

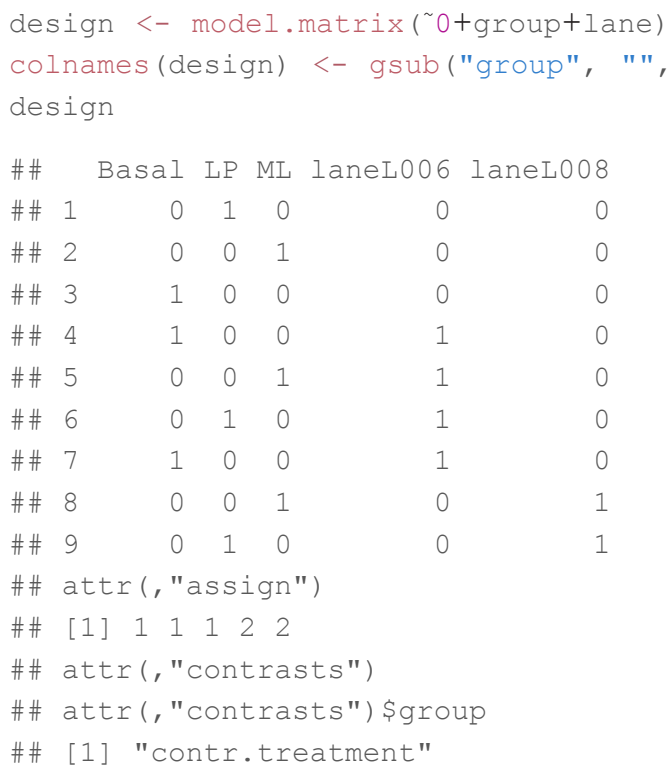


For a given experiment, there are usually several equivalent ways to set up an appropriate design matrix. For example, $\sim 0+$ group +1 ane removes the intercept from the first factor, group, but an intercept remains in the second factor lane. Alternatively, group l lane could be used to keep the intercepts in both group and lane. Understanding how to interpret the coefficients estimated in a given model is key here. We choose the first model for our analysis, as setting up model contrasts is more straight forward in the absence of an intercept for group. Contrasts for pairwise comparisons between cell populations are set up in limma using the makeContrasts function.

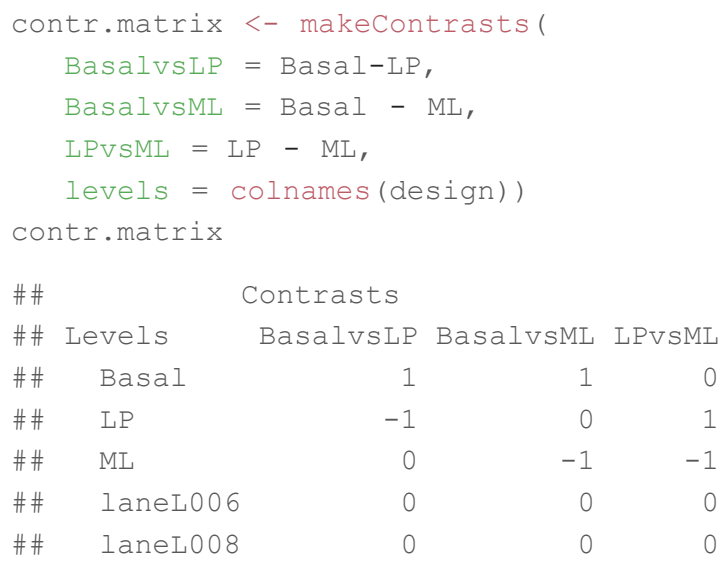

A key strength of limma's linear modelling approach is the ability accommodate arbitrary experimental complexity. Simple designs, such as the one in this workflow, with cell type and batch, through to more complicated factorial designs and models with interaction terms can be handled relatively easily. Where experimental or technical effects can be modelled using a random effect, another possibility in limma is to estimate correlations using duplicatecorrelation by specifying a block argument for both this function and in the lmFit linear modelling step.

\section{Removing heteroscedascity from count data}

It has been shown that for RNA-seq count data, the variance is not independent of the mean ${ }^{13}-$ this is true of raw counts or when transformed to log-CPM values. Methods that model counts using a Negative Binomial distribution assume a quadratic mean-variance relationship. In limma, linear modelling is carried out on the log-CPM values which are assumed to be normally distributed and the mean-variance relationship is accommodated using precision weights calculated by the voom function.

When operating on a DGEList-object, voom converts raw counts to log-CPM values by automatically extracting library sizes and normalisation factors from $\mathrm{x}$ itself. Additional normalisation to log-CPM values can be specified within voom using the normalize. method argument.

The mean-variance relationship of log-CPM values for this dataset is shown in Figure 4A. Typically, the "voom-plot" shows a decreasing trend between the means and variances resulting from a combination of technical variation in the sequencing experiment and biological variation amongst the replicate samples from different cell populations. Experiments with high biological variation usually result in flatter trends, where variance values plateau at high expression values. Experiments with low biological variation tend to result in sharp decreasing trends.

Moreover, the voom-plot provides a visual check on the level of filtering performed upstream. If filtering of lowly-expressed genes is insufficient, a drop in variance levels can be observed at the low end of the expression scale due to very small counts. If this is observed, one should return to the earlier filtering step and increase the expression threshold applied to the dataset.

Where sample-level variation is evident from earlier inspections of the MDS plot, the voomWithQualityWeights function can be used to simultaneously incorporate sample-level weights together with the abundance dependent weights estimated by voom ${ }^{14}$. For an example of this approach, see Liu et al. $(2016)^{15}$. 
A. voom: Mean-variance trend

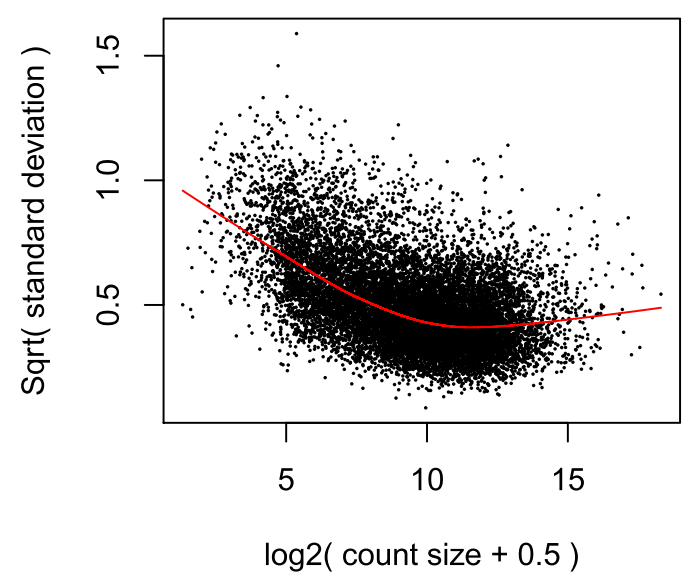

B. Final model: Mean-variance trend

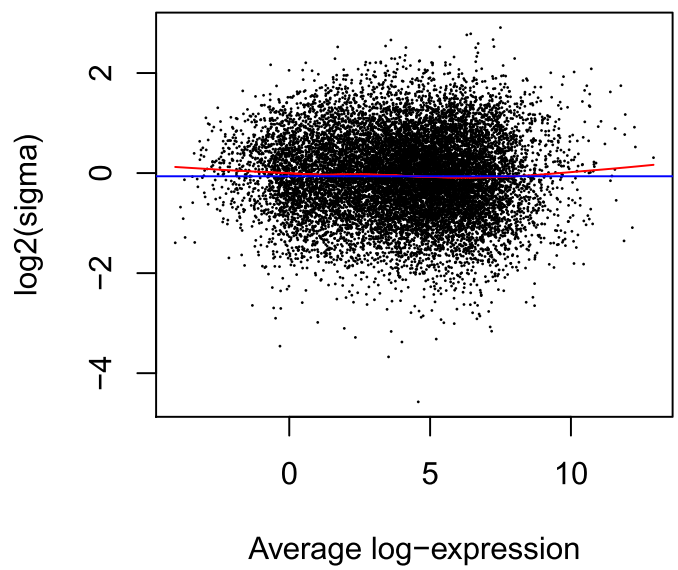

Figure 4. Means (x-axis) and variances (y-axis) of each gene are plotted to show the dependence between the two before voom is applied to the data $(\mathbf{A})$ and how the trend is removed after voom precision weights are applied to the data (B). The plot on the left is created within the voom function which extracts residual variances from fitting linear models to log-CPM transformed data. Variances are then rescaled to quarter-root variances (or square-root of standard deviations) and plotted against the mean expression of each gene. The means are $\log _{2}$-transformed mean-counts with an offset of 0.5. The plot on the right is created using plotSA which plots $\log _{2}$ residual standard deviations against mean log-CPM values. The average $\log _{2}$ residual standard deviation is marked by a horizontal blue line. In both plots, each black dot represents a gene and a red curve is fitted to these points.

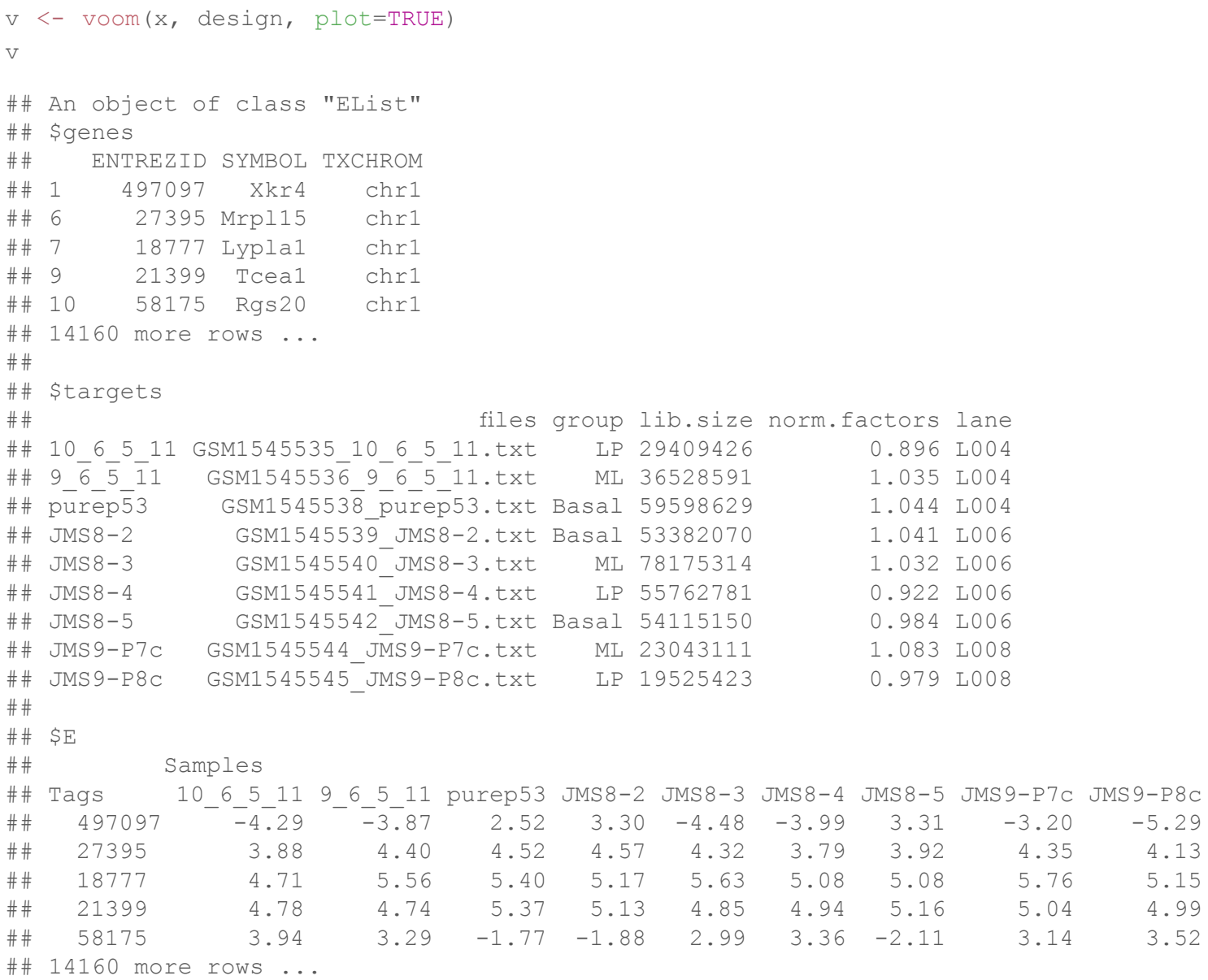




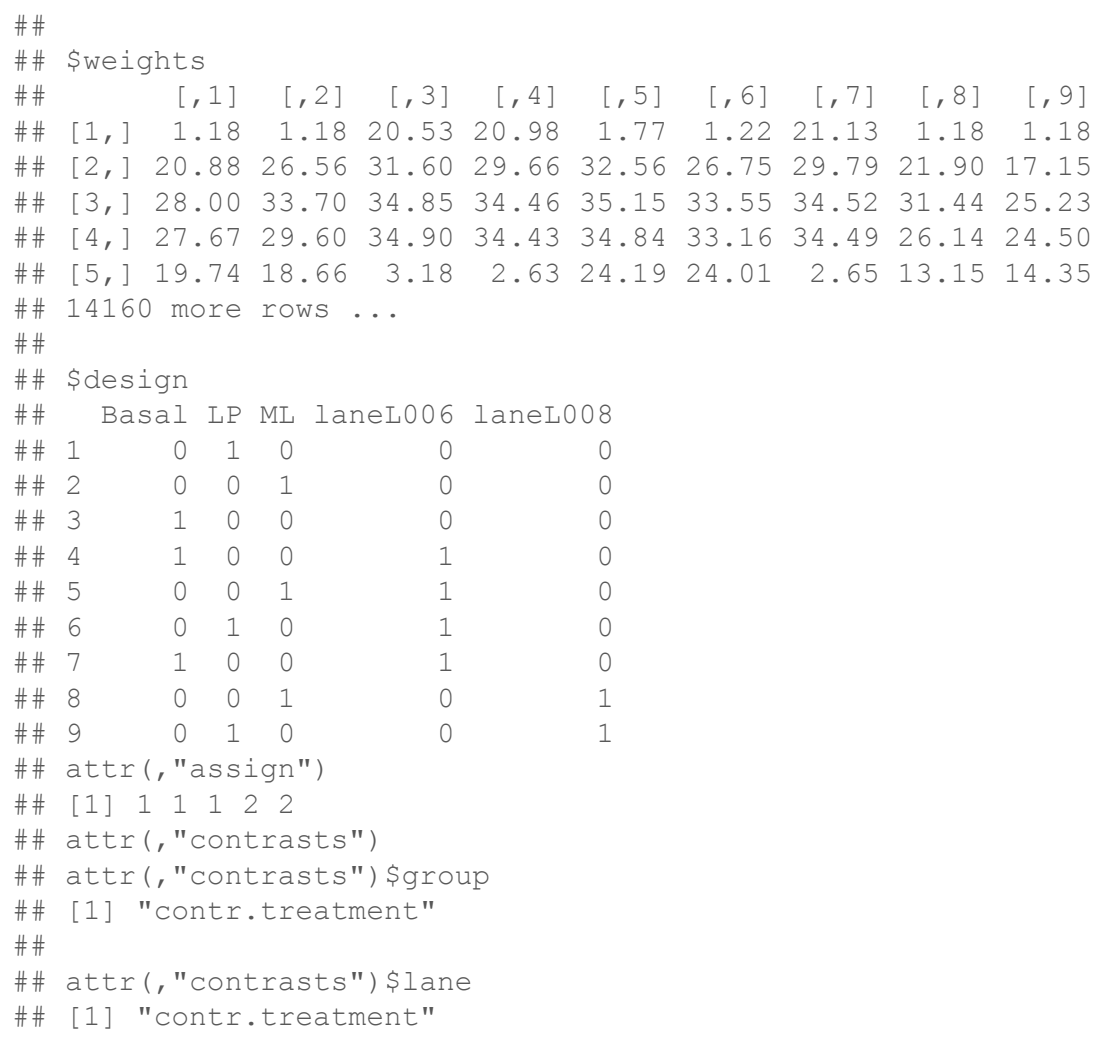

Note that the other data frames stored within the DGEList-object that contain gene- and sample-level information, are retained in the EList-object $v$ created by voom. The $v \$ g e n e s$ data frame is equivalent to $x$ \$genes, $v \$$ targets is equivalent to $x \$ s a m p l e s$, and the expression values stored in $v \$ E$ is analogous to $x \$ c o u n t s$, albeit on a transformed scale. In addition to this, the voom EList-object has a matrix of precision weights $v \$ w e i g h t s$ and stores the design matrix in $v \$$ design.

Fitting linear models for comparisons of interest

Linear modelling in limma is carried out using the lmFit and contrasts. fit functions originally written for application to microarrays. The functions can be used for both microarray and RNA-seq data and fit a separate model to the expression values for each gene. Next, empirical Bayes moderation is carried out by borrowing information across all genes to obtain more precise estimates of gene-wise variability ${ }^{16}$. The model's residual variances are plotted against average expression values in Figure 4B. It can be seen from this plot that the variance is no longer dependent on the mean expression level.

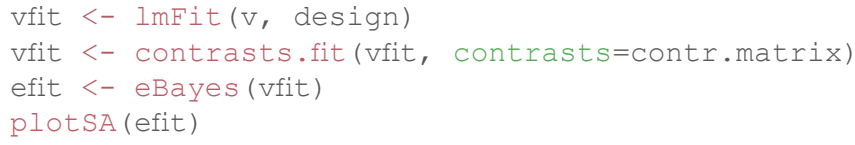

\section{Examining the number of DE genes}

For a quick look at differential expression levels, the number of significantly up- and down-regulated genes can be summarised in a table. Significance is defined using an adjusted $p$-value cutoff that is set at $5 \%$ by default. For the comparison between expression levels in basal and LP, 4,127 genes are found to be down-regulated in basal relative to LP and 4,298 genes are up-regulated in basal relative to LP - a total of 8,425 DE genes. A total of 8,510 DE genes are found between basal and ML (4,338 down- and 4,172 up-regulated genes), and a total of 5,340 DE genes are found between LP and ML (2,895 down- and 2,445 up-regulated). The larger numbers of DE genes observed for comparisons involving the basal population are consistent with our observations from the MDS plots. 


\begin{tabular}{|c|c|c|c|}
\hline \#\# \# & BasalvsLP & BasalvsML & LPVsM \\
\hline$\#-1$ & 4127 & 4338 & 289 \\
\hline$\# 0$ & 5740 & 5655 & 882 \\
\hline 1 & 298 & 4172 & \\
\hline
\end{tabular}

Some studies require more than an adjusted $p$-value cutoff. For a stricter definition on significance, one may require log-fold-changes (log-FCs) to be above a minimum value. The treat method ${ }^{17}$ can be used to calculate $p$-values from empirical Bayes moderated $t$-statistics with a minimum log-FC requirement. The number of differentially expressed genes are reduced to a total of 3,135 DE genes for basal versus LP, 3,270 DE genes for basal versus ML, and $385 \mathrm{DE}$ genes for $\mathrm{LP}$ versus ML when testing requires genes to have a log-FC that is significantly greater than 1 (equivalent to a 2-fold difference between cell types on the original scale).

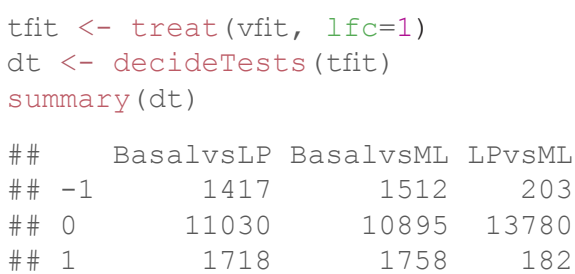

Genes that are DE in multiple comparisons can be extracted using the results from decideTests, where 0s represent genes that are not DE, 1s represent genes that are up-regulated, and $-1 \mathrm{~s}$ represent genes that are down-regulated. A total of 2,409 genes are DE in both basal versus LP and basal versus ML (Figure 5), twenty of which are listed below. The write. fit function can be used to extract and write results for all three comparisons to a single output file.
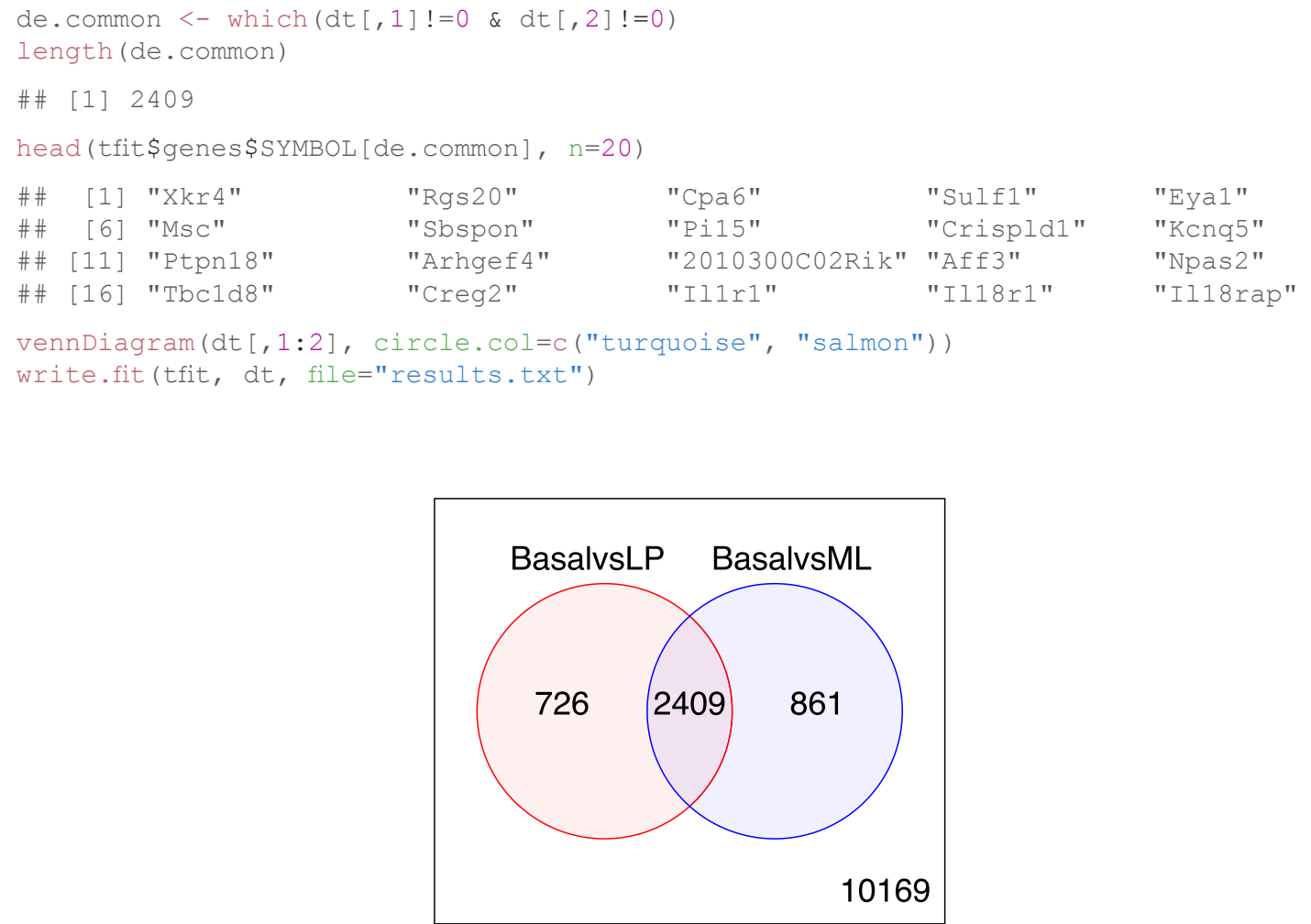

Figure 5. Venn diagram showing the number of genes DE in the comparison between basal versus LP only (left), basal versus ML only (right), and the number of genes that are $\mathrm{DE}$ in both comparisons (center). The number of genes that are not DE in either comparison are marked in the bottom-right. 
Examining individual DE genes from top to bottom

The top DE genes can be listed using topTreat for results using treat (or topTable for results using eBayes). By default topTreat arranges genes from smallest to largest adjusted $p$-value with associated gene information, $\log$-FC, average $\log$-CPM, moderated $t$-statistic, raw and adjusted $p$-value for each gene. The number of top genes displayed can be specified, where $n=\operatorname{Inf}$ includes all genes. Genes $\mathrm{Cldn} 7$ and Rasef are amongst the top DE genes for both basal versus LP and basal versus ML.

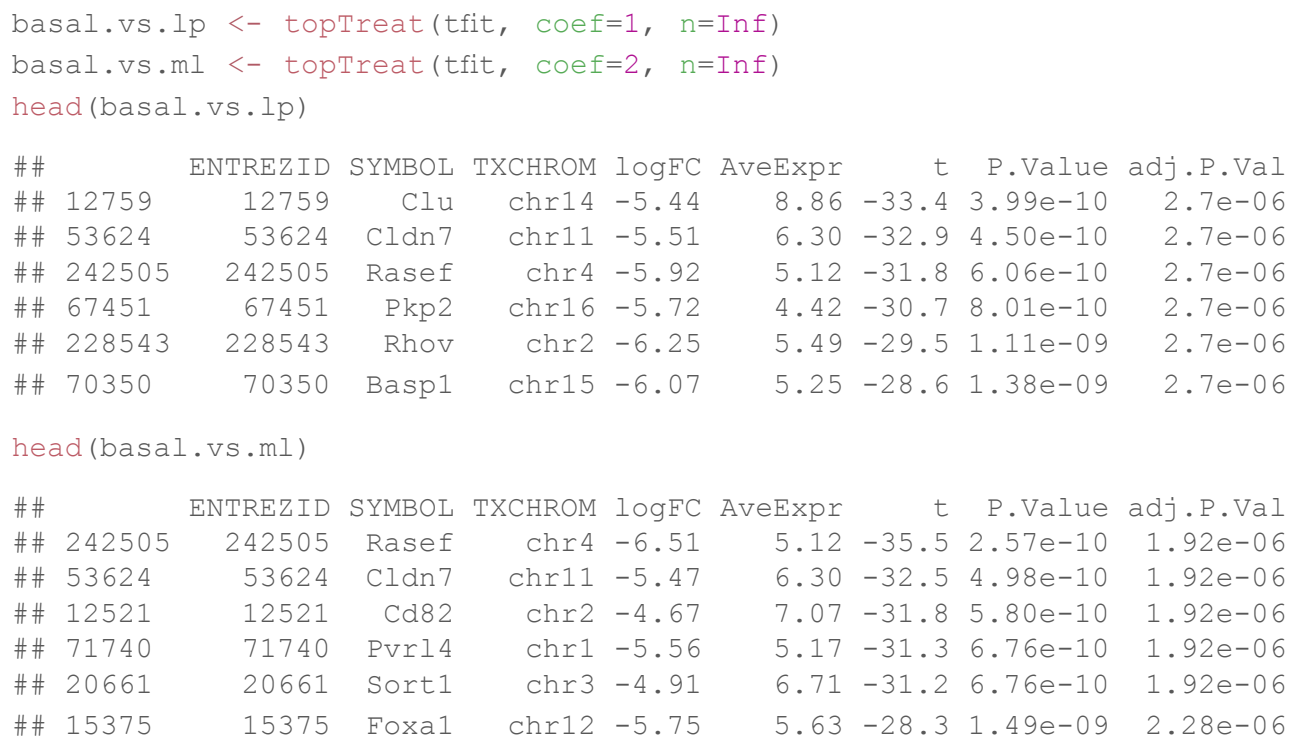

Useful graphical representations of differential expression results

To summarise results for all genes visually, mean-difference plots, which display log-FCs from the linear model fit against the average log-CPM values can be generated using the plotMD function, with the differentially expressed genes highlighted.

plotmD (tfit, column=1, status=dt[,1], main=colnames (tfit) $[1], x \lim =c(-8,13)$ )

Glimma extends this functionality by providing an interactive mean-difference plot via the glMDPlot function. The output of this function is an html page, with summarised results in the left panel (similar to what is output by plotMD), and the log-CPM values from individual samples for a selected gene in the right panel, with a table of results below the plots (Figure 6). This interactive display allows the user to search for particular genes based on the annotation provided (e.g. Gene symbol identifier), which is not possible in a static R plot.

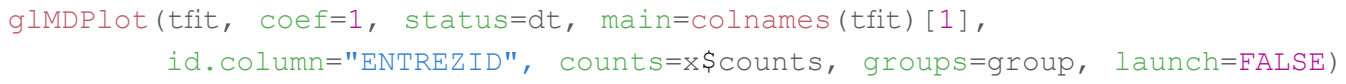

The mean-difference plot generated by the command above is available online (see http://bioinf.wehi.edu.au/folders/ limmaWorkflow/glimma-plots/MD-Plot.html). The interactivity provided by the Glimma package allows additional information to be presented in a single graphical window. Glimma is implemented in R and Javascript, with the $R$ code generating the data which is converted into graphics using the Javascript library D3 (https://d3js.org), with the Bootstrap library handling layouts and Datatables generating the interactive searchable tables. This allows plots to be viewed in any modern browser, which is convenient for including them as linked files from an Rmarkdown report of the analysis.

Plots shown previously include either all of the genes that are expressed in any one condition (such as the Venn diagram of common DE genes or mean-difference plot) or look at genes individually (log-CPM values shown in right panel of the interactive mean-difference plot). Heatmaps allow users to look at the expression of a subset of genes. This can give useful insight into the expression of individual groups and samples without losing perspective of the overall study when focusing on individual genes, or losing resolution when examining patterns averaged over thousands of genes at the same time. 
BasalvsLP

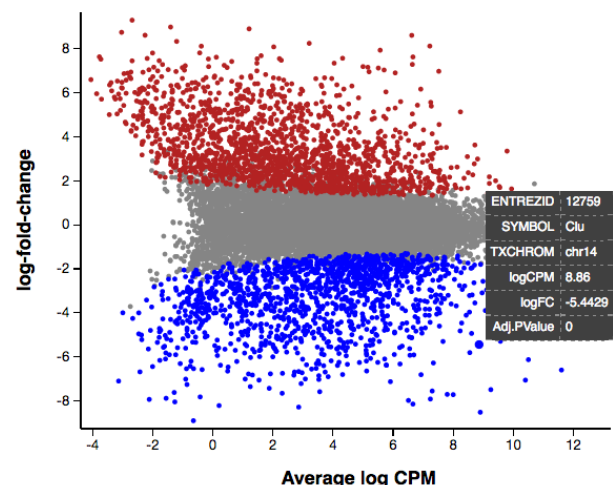

Show 10 entries

ENTREZID $\triangle$ SYMBOL

\begin{tabular}{|l|l|l|}
\hline 12759 & Clu & chr14 \\
\hline 74148 & Cluh & chr11 \\
\hline 76779 & Cluap1 & chr16 \\
\hline
\end{tabular}

Showing 1 to 3 of 3 entries (filtered from 14,165 total entries)1 row selected
12759

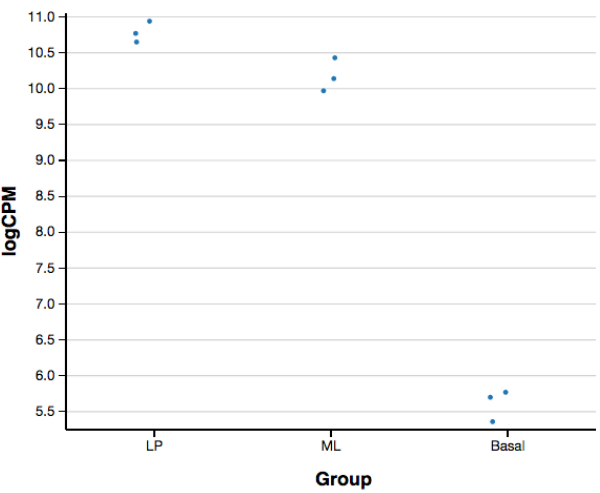

Search: Clu

\begin{tabular}{|l|l|l|}
\hline 8.86 & -5.442877 & 0.0000027 \\
\hline 6.82 & 0.208553 & 1 \\
\hline 4.01 & 0.148665 & 1 \\
\hline
\end{tabular}

Figure 6. Interactive mean-difference plot generated using Glimma. Summary data (log-FCs versus log-CPM values) are shown in the left panel which is linked to the individual values per sample for a selected gene in the right panel. A table of results is also displayed below these figures, along with a search bar to allow users to look up a particular gene using the annotation information available, e.g. the Gene symbol identifier Clu.

A heatmap is created for the top $100 \mathrm{DE}$ genes (as ranked by adjusted $p$-value) from the basal versus LP contrast using the heatmap. 2 function from the gplots package (Figure 7). The heatmap correctly clusters samples by cell type and reorders the genes into blocks with similar expression patterns. From the heatmap, we observe that the expression of ML and LP samples are very similar for the top $100 \mathrm{DE}$ genes between basal and LP.

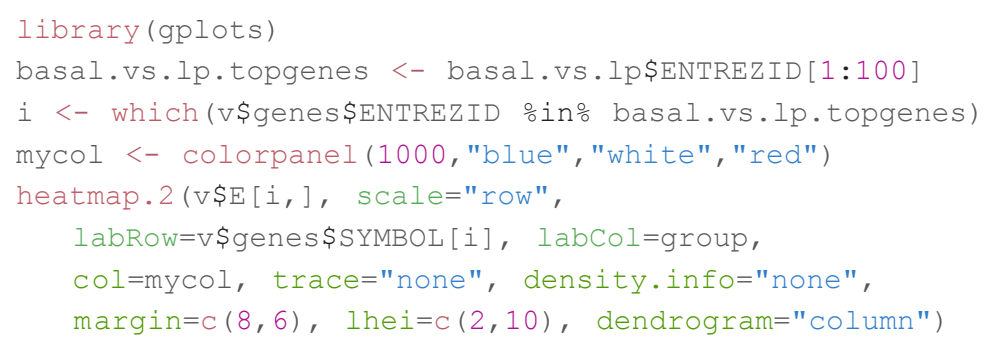

\section{Gene set testing with camera}

We finish off this analysis with some gene set testing by applying the camera method $^{18}$ to the $c 2$ gene signatures from the Broad Institute's MSigDB c2 collection ${ }^{19}$ that have been adapted for mouse and are available as Rdata objects from http://bioinf.wehi.edu.au/software/MSigDB/. Other useful gene sets derived from MSigDB for both human and mouse, such as the hallmark gene sets, are also available from this site. C2 gene sets have been curated from online databases, publications and domain experts, and hallmark gene sets are selected from MSigDB to represent well-defined biological states or processes. 

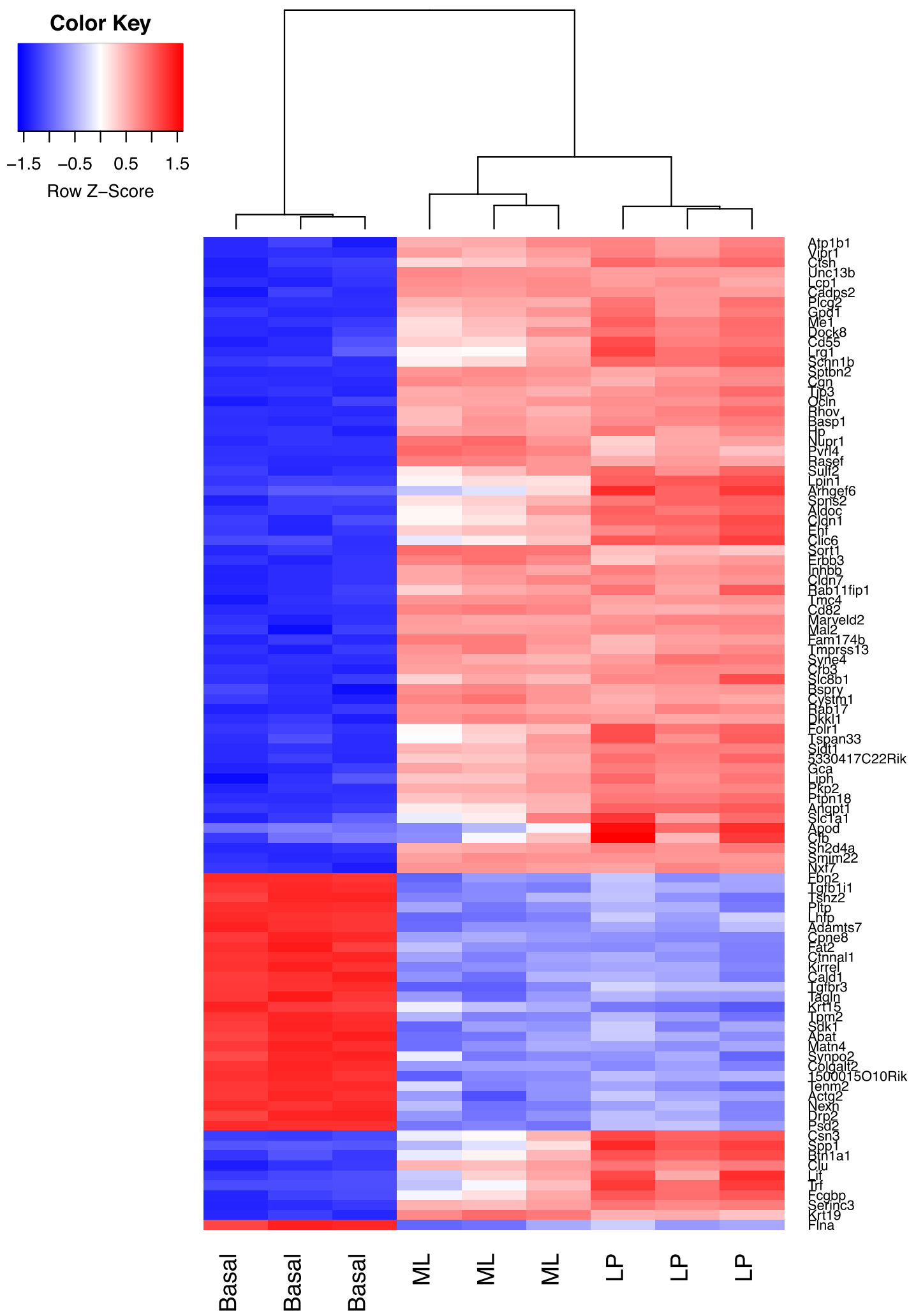

Figure 7. Heatmap of log-CPM values for top 100 genes DE in basal versus LP. Expression across each gene (or row) have been scaled so that mean expression is zero and standard deviation is one. Samples with relatively high expression of a given gene are marked in red and samples with relatively low expression are marked in blue. Lighter shades and white represent genes with intermediate expression levels. Samples and genes have been reordered by the method of hierarchical clustering. A dendrogram is shown for the sample clustering. 


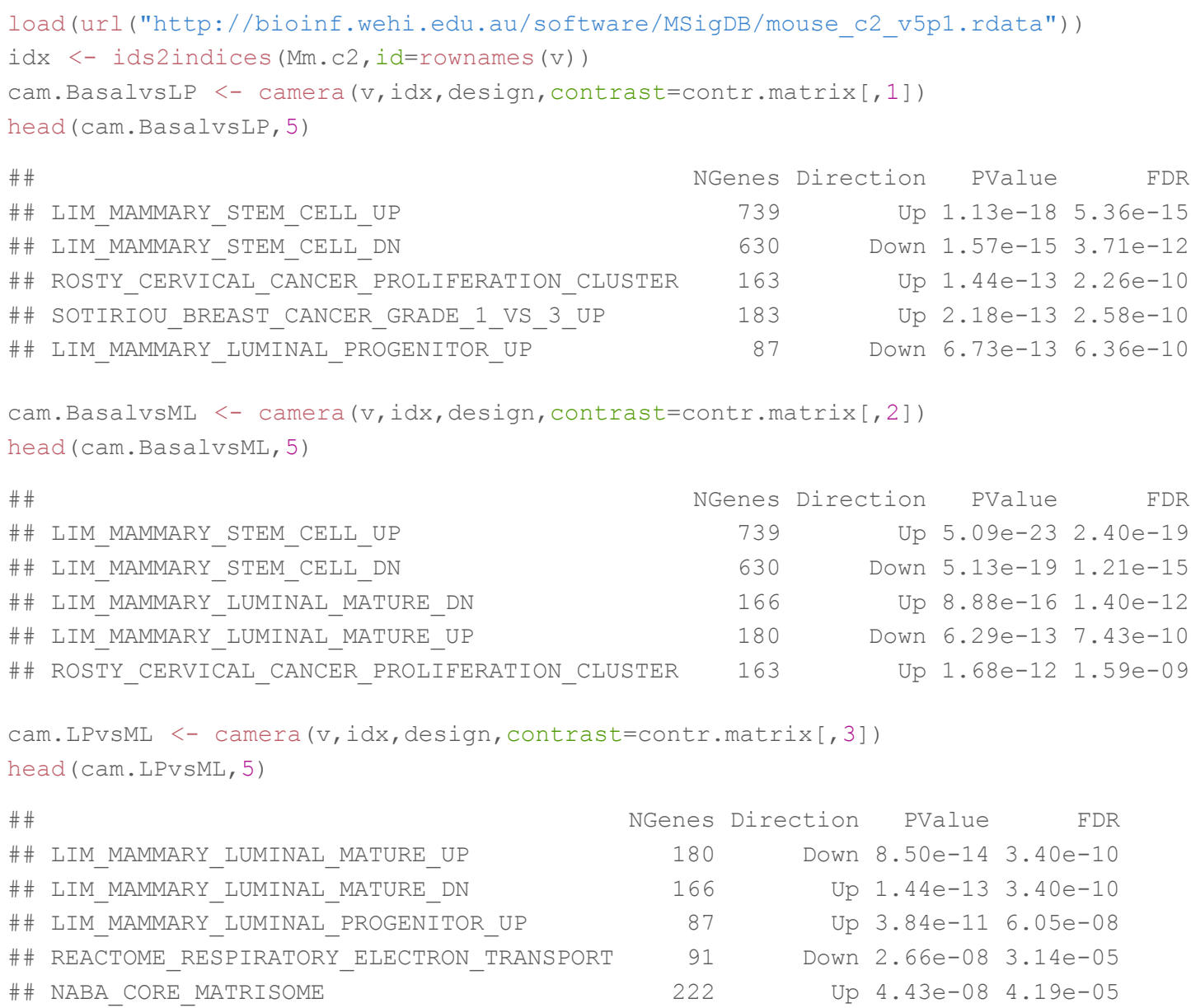

The camera function performs a competitive test to assess whether the genes in a given set are highly ranked in terms of differential expression relative to genes that are not in the set. It uses limma's linear model framework, taking both the design matrix and contrast matrix (if present) and accommodates the observational-level weights from voom in the testing procedure. After adjusting the variance of the resulting gene set test statistic by a variance inflation factor that depends on the gene-wise correlation (which is set to 0.01 by default, but can be estimated from the data) and the size of the set, a $p$-value is returned and adjusted for multiple testing.

This experiment is the RNA-seq equivalent of the dataset generated by Lim et al. (2010) ${ }^{20}$, who used Illumina microarrays to profile the same sorted cell populations, so it is reassuring to see the gene signatures from this earlier publication coming up at the top of the list for each contrast. We make a barcodeplot of the Lim et al. (2010) Mature Luminal gene sets (Up and Down) in the LP versus ML contrast. Note that these sets go in the opposite direction in our dataset due to our parameterization which compares LP against ML rather than the other way around (if the contrast were reversed, the directions would be consistent).

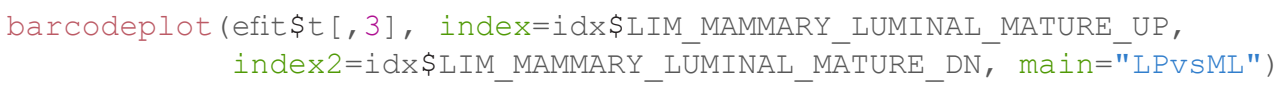

Other gene set tests are available in limma, such as the self-contained tests by mroast ${ }^{21}$. Whilst camera is ideal for testing a large database of gene sets and observing which of them rank highly relative to others (as shown above), selfcontained tests are better for focused testing of one or a few specifically chosen sets to see if they are DE in their own right. In other words, camera is more appropriate when "fishing" for gene sets of interest, whereas mroast tests sets that are already of interest for significance. 


\section{LPvsML}

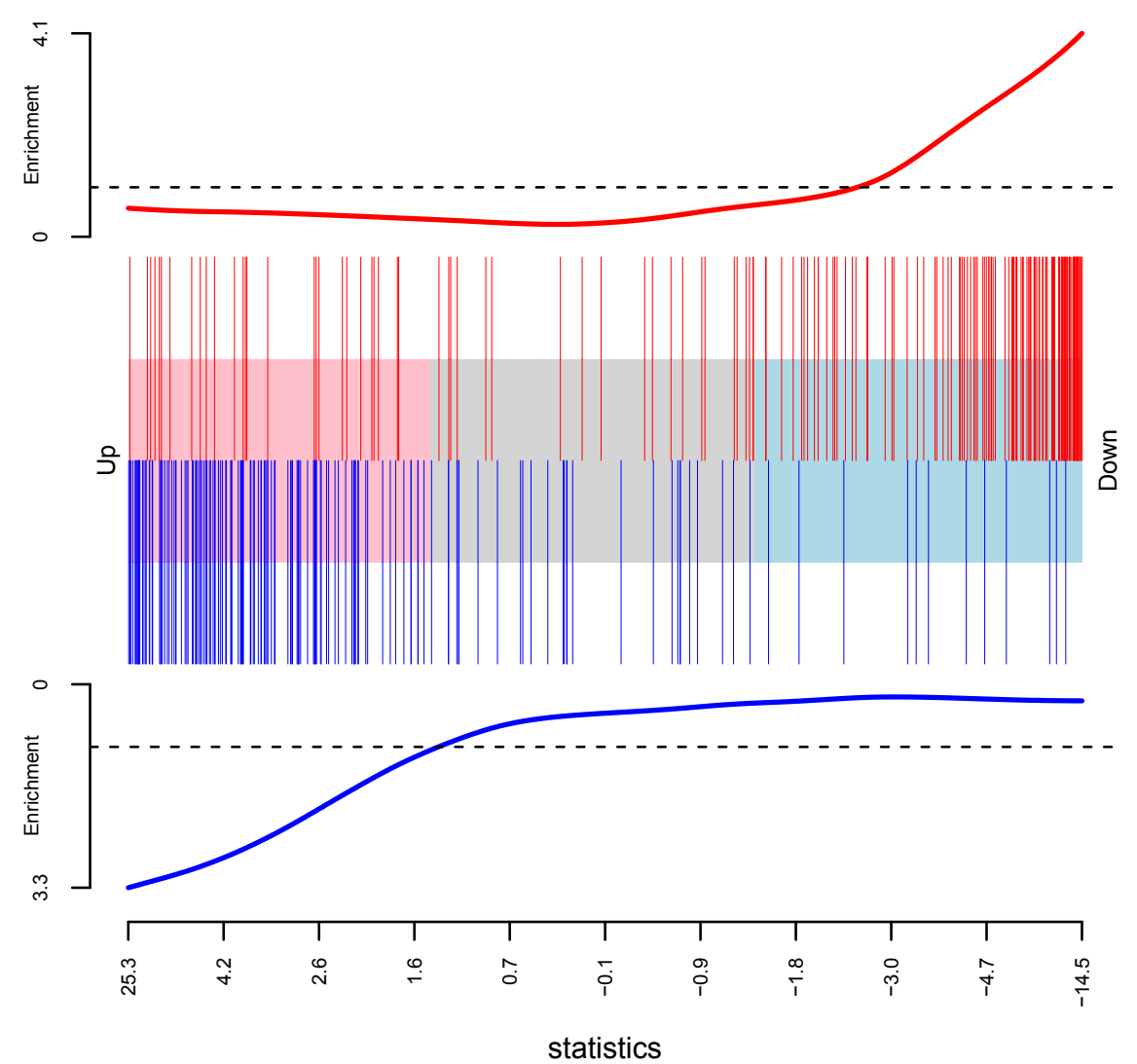

Figure 8. Barcode plot of LIM_MAMMARY_LUMINAL_MATURE_UP (red bars, top of plot) and LIM_MAMMARY LUMINAL_MATURE_DN (blue bars, bottom of plot) gene sets in the LP versus ML contrast. For each set, an enrichment line that shows the relative enrichment of the vertical bars in each part of the plot is displayed. The experiment of Lim et al. (2010) is very similar to the current one, with the same sorting strategy used to obtain the different cell populations, except that microarrays were used instead of RNA-seq to profile gene expression. Note that the inverse correlation (the up gene set is down and the down gene set is up) is a result of the way the contrast has been set up (LP versus ML) - if reversed, the directionality would agree.

\section{Software availability}

This RNA-seq workflow makes use of various packages available from version 3.4 of the Bioconductor project, running on $\mathrm{R}^{22}$ version 3.3.0 or higher. Besides the software highlighted in this article (limma, Glimma and edgeR) it requires a number of other packages, including gplots $^{23}$ and RColorBrewer and the gene annotation package Mus.musculus. This document was compiled using knitr $^{24-26}$. Version numbers for all packages used are shown below. Code to perform this analysis is available in the Bioconductor workflow package RNAseq123 from http://www.bioconductor.org/help/workflows/RNAseq123/.

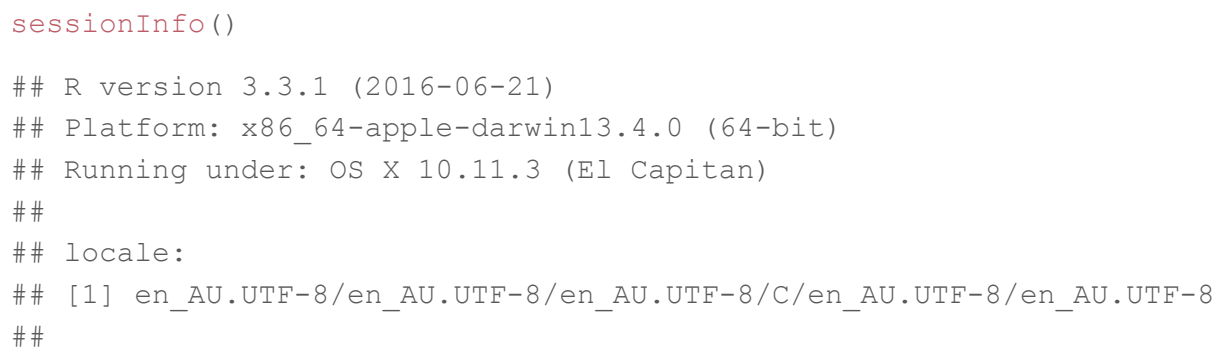




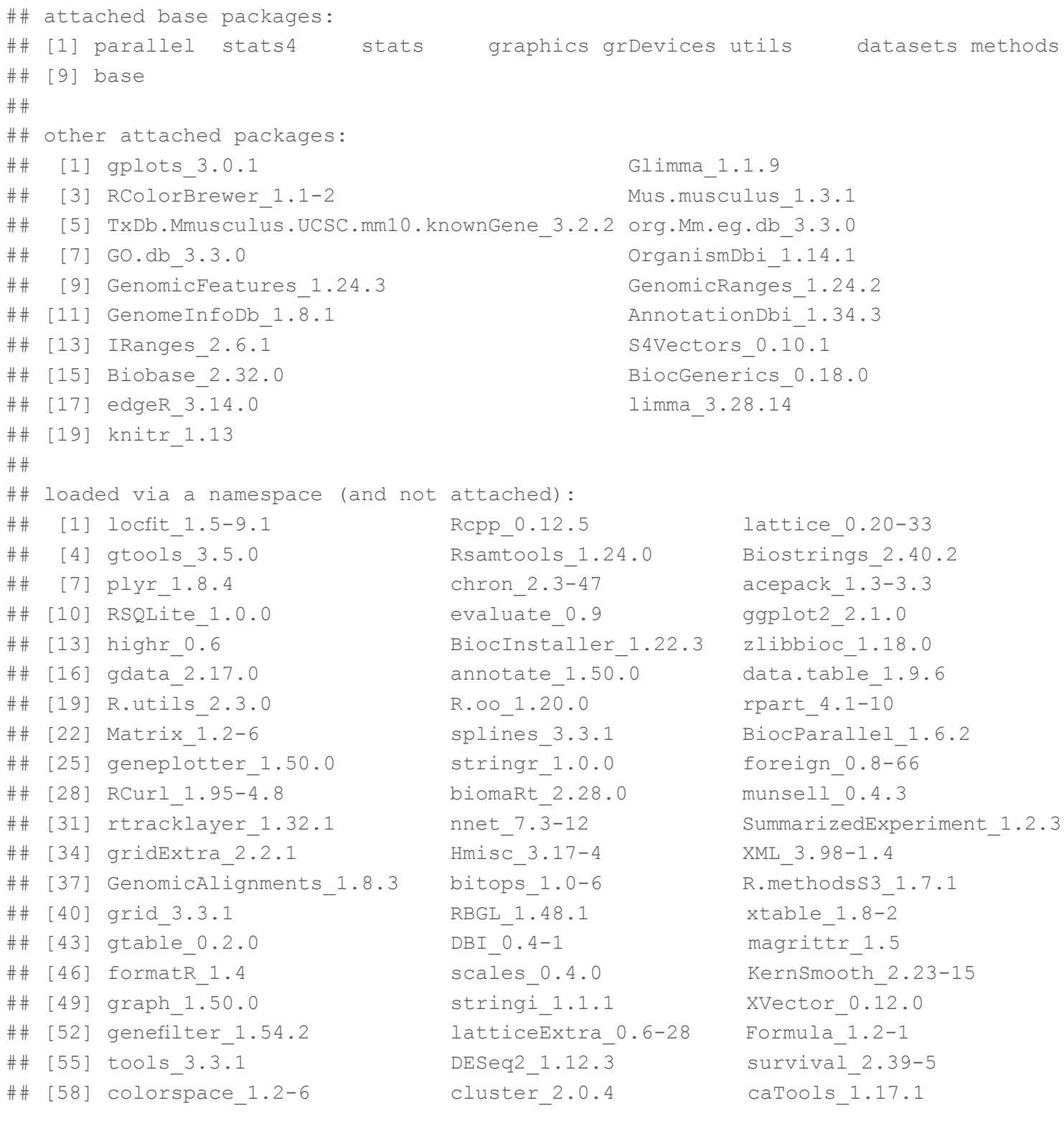

\section{Author contributions}

CWL and MER developed the workflow and wrote the article with input from MA (gene set testing), SS (Glimma) and GKS (general editing and advice on direction of the article).

\section{Competing interests}

The authors declare that they have no competing interests. 
Grant information

This worked was funded by the National Health and Medical Research Council (NHMRC) (Fellowship GNT1058892 and Program GNT1054618 to GKS, Project GNT1050661 to MER and GKS and Fellowship GNT1104924 to MER), Victorian State Government Operational Infrastructure Support and Australian Government NHMRC IRIISS.

The funders had no role in study design, data collection and analysis, decision to publish, or preparation of the manuscript.

\section{Acknowledgements}

We thank Dr Julie Sheridan for generating this dataset and for advice on its analysis.

1. Robinson MD, McCarthy DJ, Smyth GK: edgeR: a Bioconductor package for differential expression analysis of digital gene expression data. Bioinformatics. 2010; 26(1): 139-140. PubMed Abstract | Publisher Full Text | Free Full Text

2. Ritchie ME, Phipson B, Wu D, et al.: limma powers differential expression analyses for RNA-sequencing and microarray studies. Nucleic Acids Res. 2015; 43(7): e47.

PubMed Abstract | Publisher Full Text | Free Full Text

3. Huber W, Carey VJ, Gentleman R, et al.: Orchestrating highthroughput genomic analysis with Bioconductor. Nat Methods. 2015; 12(2): 115-121.

PubMed Abstract | Publisher Full Text | Free Full Text

4. Su S, Ritchie ME: Glimma: Interactive HTML graphics for RNA-seq data. 2016; $R$ package version 1.1.1

5. Sheridan JM, Ritchie ME, Best SA, et al.: A pooled shRNA screen for regulators of primary mammary stem and progenitor cells identifies roles for Asap1 and Prox1. BMC Cancer. 2015; 15(1): 221.

PubMed Abstract | Publisher Full Text | Free Full Text

6. Liao Y, Smyth GK, Shi W: The Subread aligner: fast, accurate and scalable read mapping by seed-and-vote. Nucleic Acids Res. 2013, 41(10): e108.

PubMed Abstract | Publisher Full Text | Free Full Text

7. Liao Y, Smyth GK, Shi W: featureCounts: an efficient general purpose program for assigning sequence reads to genomic features. Bioinformatics. 2014; 30(7): 923-30.

PubMed Abstract | Publisher Full Text

8. Bioconductor Core Team: Mus.musculus: Annotation package for the Mus.musculus object. 2016; R package version 1.3.1. Reference Source

9. Bioconductor Core Team: Homo.sapiens: Annotation package for the Homo.sapiens object. 2016; R package version 1.3.1. Reference Source

10. Durinck S, Moreau Y, Kasprzyk A, et al.: BioMart and Bioconductor: a powerful link between biological databases and microarray data analysis. Bioinformatics. 2005; 21(16): 3439-40. PubMed Abstract | Publisher Full Text

11. Durinck S, Spellman PT, Birney E, et al:: Mapping identifiers for the integration of genomic datasets with the R/Bioconductor package biomaRt. Nat Protoc. 2009; 4(8): 1184-91. PubMed Abstract | Publisher Full Text | Free Full Text

12. Robinson $M D$, Oshlack $A$ : A scaling normalization method for differential expression analysis of RNA-seq data. Genome Biol. 2010; 11(3): R25.

PubMed Abstract | Publisher Full Text | Free Full Text

13. Law CW, Chen $\mathrm{Y}$, Shi W, et al.: voom: Precision weights unlock linear model analysis tools for RNA-seq read counts. Genome Biol. 2014; 15(2): R29.

PubMed Abstract | Publisher Full Text | Free Full Text
14. Liu R, Holik AZ, Su S, et al:: Why weight? Modelling sample and observational level variability improves power in RNA-seq analyses. Nucleic Acids Res. 2015; 43(15): e97. PubMed Abstract | Publisher Full Text | Free Full Text

15. Liu R, Chen K, Jansz N, et al.: Transcriptional profiling of the epigenetic regulator Smchd1. Genom Data. 2016; 7: 144-7. PubMed Abstract | Publisher Full Text | Free Full Text

16. Smyth GK: Linear models and empirical bayes methods for assessing differential expression in microarray experiments. Stat Appl Genet Mol Biol. 2004; 3(1): Article3. PubMed Abstract | Publisher Full Text

17. McCarthy DJ, Smyth GK: Testing significance relative to a foldchange threshold is a TREAT. Bioinformatics. 2009; 25(6): 765-71. PubMed Abstract | Publisher Full Text | Free Full Text

18. Wu D, Smyth GK: Camera: a competitive gene set test accounting for inter-gene correlation. Nucleic Acids Res. 2012; 40(17): e133. PubMed Abstract | Publisher Full Text | Free Full Text

19. Subramanian A, Tamayo P, Mootha VK, et al:: Gene set enrichment analysis: a knowledge-based approach for interpreting genomewide expression profiles. Proc Natl Acad Sci U S A. 2005; 102(43): $15545-50$.

PubMed Abstract | Publisher Full Text | Free Full Text

20. Lim E, Wu D, Pal B, et al.: Transcriptome analyses of mouse and human mammary cell subpopulations reveal multiple conserved genes and pathways. Breast Cancer Res. 2010; 12(2): R21. PubMed Abstract | Publisher Full Text | Free Full Text

21. Wu D, Lim F, Vaillant F, et al.: ROAST: rotation gene set tests for complex microarray experiments. Bioinformatics. 2010; 26(17): 2176-82.

PubMed Abstract | Publisher Full Text | Free Full Text

22. R Development Core Team: R: A language and environment for statistical computing. R Foundation for Statistical Computing, Vienna, 2016.

Reference Source

23. Warnes GR, Bolker B, Bonebakker L, et al:: gplots: Various $\mathbf{R}$ Programming Tools for Plotting Data. 2016; R package version 3.0.1.

Reference Source

24. Xie Y: knitr: A comprehensive tool for reproducible research in R. In: V. Stodden, F. Leisch, and R. D. Peng, editors, Implementing Reproducible Computational Research. Chapman and Hall/CRC, 2014; ISBN 978-1466561595 Reference Source

25. Xie Y: Dynamic Documents with $\mathbf{R}$ and knitr. Chapman and Hall/ CRC, Boca Raton, Florida, 2nd edition, 2015; ISBN 978-1498716963. Reference Source

26. Xie Y: knitr: A General-Purpose Package for Dynamic Report Generation in R. 2016; R package version 1.12.3. Reference Source 


\title{
Open Peer Review
}

\section{Current Peer Review Status:}

\section{Version 1}

Reviewer Report 06 July 2016

https://doi.org/10.5256/f1000research.9688.r14437

(C) 2016 Maksimovic J. This is an open access peer review report distributed under the terms of the Creative Commons Attribution License, which permits unrestricted use, distribution, and reproduction in any medium, provided the original work is properly cited.

\author{
Jovana Maksimovic \\ 1 Bioinformatics research group, Murdoch Childrens Research Institute, Murdoch Children's \\ Research Institute, Parkville, Vic, Australia \\ 2 Bioinformatics research group, Murdoch Childrens Research Institute, Murdoch Children's \\ Research Institute, Parkville, Vic, Australia
}

This workflow outlines a step-by-step differential expression analysis of a publicly available RNAseq dataset using several well-established Bioconductor packages such as limma and edgeR and the novel Glimma package. The workflow describes in detail the key steps that are generally performed as part of most standard, count-based RNAseq analyses: pre-processing, exploratory data analysis, differential expression testing and pathway analysis. Each of the steps is carefully explained and broken down into a series of logical substeps, which also include R code and example output. Furthermore, this workflow is a good example of how multiple Bioconductor packages can be applied in a real-world RNAseq analysis.

Overall, I think this is a very clearly written and well-explained workflow that not only highlights the utility of combining Bioconductor packages for RNAseq analysis but also demystifies the process for those who may be interested in learning what an RNAseq analysis involves. Although some familiarity with R and statistical concepts would be advantageous, they are not necessary to be able to follow the logic behind the steps and what each step is trying to achieve.

I have tested the R code under R 3.3.0 and Bioconductor 3.0 and it ran without errors.

\section{Minor comments:}

As this workflow will be of particular use for those who are very new to RNAseq analysis, I think it should be explained why 1 CPM is chosen as the cutoff for lowly expressed genes. In addition, it may be useful to show the relationship between CPM and raw counts and how the selected cutoff relates to numbers of reads.

In the gene set testing section, it is worth mentioning that the Hallmark gene sets (also available from the Broad MSigDB) may be a good starting point for pathway analysis as they "summarise and represent specific well-defined, states or processes". 
Also, for those who are new to RNAseq analysis, it may be worth mentioning that there are other gene set testing methods available in limma e.g. roast etc. and a very brief explanation of when the different methods are appropriate.

General comment regarding Glimma plots: it would be handy to see the gene symbol (if available) as well as EntrezID in the title of the scatter plot next to the MD plot.

Competing Interests: Matthew E. Ritchie and I are both organisers of the Bioconductor Asia-Pacific Developer meeting, which is to be held in Brisbane in November 2016.

I confirm that I have read this submission and believe that I have an appropriate level of expertise to confirm that it is of an acceptable scientific standard.

Author Response 30 Nov 2016

Charity Law

Dear Jovana, thank-you for the helpful suggestions. We have added extra text to explain why a CPM value of 1 was chosen and how this value relates to raw counts. We have also given more detail on $\mathrm{c} 2$ and Hallmark gene sets, as well as mentioning roast and when one might chose to use roast over camera.

Competing Interests: None

Reviewer Report 06 July 2016

https://doi.org/10.5256/f1000research.9688.r14439

(C) 2016 Doyle M. This is an open access peer review report distributed under the terms of the Creative Commons Attribution License, which permits unrestricted use, distribution, and reproduction in any medium, provided the original work is properly cited.

\section{Maria A. Doyle}

${ }^{1}$ Research Computing Facility, Peter MacCallum Cancer Centre, Melbourne, Vic, Australia

2 Research Computing Facility, Peter MacCallum Cancer Centre, Melbourne, Vic, Australia

Editorial Note from F1000Research - 7th July 2016:

the status of this review was changed after publication from "Approved with Reservations" to "Approved". The referee's reservation was caused by using the current release (Bioconductor 3.3) as opposed to the development release (Bioconductor 3.4) as used in the article.

The following text has been removed:

My only reservation to approving the article is that I encountered these two errors when running the Glimma code in the article. I used R 3.3.0 and the most recent version of Glimma 


\section{from Bioconductor (Glimma 1.0.0) but the sessionInfo() in the article shows Glimma 1.1.1 was used which may account for the errors:}

1) With this line of code no plot was produced and it gave the error below: gIMDSPlot(Icpm, labels=paste(group, lane, sep="_"), $+\quad$ groups=x\$samples[,c $(2,5)]$, launch=TRUE)

Error in glScatter.default(points, xval = "dim1", yval = "dim2", xlab = "Dimension 1", : group does not correspond to a column

2) With this line of code the table (shown in Fig 6.) didn't appear, just the 2 plots:

gIMDPlot(tfit, coef=1, status=dt[,1], main=colnames(tfit)[1], counts $=x \$$ counts, samples=colnames $(x)$, anno=x $\$$ genes, groups=group, id.column="ENTREZID", display.columns=c("SYMBOL", "ENTREZID"), search.by="SYMBOL", launch=FALSE)

This article is a very nice description (nicely written, nicely explained) of an RNA-seq workflow, starting from gene counts and proceeding through a standard differential expression analysis, using 3 complementary Bioconductor packages edgeR, limma \& Glimma. It explains clearly how to perform the steps of a differential expression analysis and also how to generate useful plots for visualising the data e.g. MDSplot, MDplot, barcodeplot, heatmap etc.

Two of the packages, edgeR and limma, are well established while Glimma is the new kid on the block. It's a tool along the lines of the web-based Degust

(http://www.vicbioinformatics.com/degust/), in that it enables interactive exploration of RNA-seq data. It would seem to be a very useful addition to R RNA-seq workflows as the interactive html plots it can generate (MDS, MD etc) make exploring the results easier (especially by non-R savvy collaborators) and it could help save an analyst's time and effort through not having to reproduce static plots e.g. to highlight different genes. With the Glimma MDplot I like that you can search for a gene and see a plot of the log-cpm counts for that gene in the samples.

It was great to be able to try out the workflow really easily by downloading the data file linked to in the paper (from GEO), with no processing required other than unzipping the files, and the code in the article all worked, giving the same results shown in the paper with the exception of two errors described below.

Minor comments:

The article says the workflow is available from Bioconductor here http://www.bioconductor.org/help/workflows/ but it doesn't seem to be there, however it is available at the other location mentioned http://bioinf.wehi.edu.au/folders/limmaWorkflow/

The section title "Removing genes that are not expressed" suggests only genes with no expression are removed, however the paragraph then explains that genes lowly expressed are also removed so for greater clarity maybe that could be changed to something like "Removing genes that are not sufficiently expressed".

In the section "Organising gene annotations" I think it's good the authors point out to check for duplicated genes, however in this case the duplication appears to be due to extracting the 
TXCHROM column (as some genes are reported as being present on more than one chromosome) but the TXCHROM information is not used in this workflow and if the TXCHROM column is omitted then there are no duplicates so it might be worth mentioning that.

With this bit "Differential expression analyses look at gene expression differences between conditions, rather than comparing expression across multiple genes or drawing conclusions on absolute levels of expression. In other words, gene lengths remain constant for comparisons of interest and any observed differences are a result of changes in condition rather than changes in gene length." Wouldn't one caveat to this be if there was a significant change in the length of the isoform(s) expressed from a gene (e.g. from expression of a long isoform to a short isoform) as then the assumption of no change in gene length would no longer be valid.

In the online version Fig. 7 is in the middle of a code block could it be moved below.

Figs 5-8 have bold headings but Figs 1-4 don't is that an error.

Could perhaps modify title to reflect the order in which the tools are used - edger, limma \& Glimma.

It would be nice for consistency if the colours in the Glimma interactive MDS plots matched the colours used in the static MDS plots in Fig 3.

Competing Interests: No competing interests were disclosed.

\section{I confirm that I have read this submission and believe that I have an appropriate level of expertise to confirm that it is of an acceptable scientific standard.}

Reviewer Response 06 Jul 2016

\section{Maria A. Doyle}

I've just requested that the status of this article be changed to Approved as the code works fine with the development release of Bioconductor 3.4 but could it be made clearer in the article that 3.4 must be used for the code to work as shown.

Competing Interests: No competing interests were disclosed.

Author Response 30 Nov 2016

\section{Charity Law}

Dear Maria, we have made changes according to your suggestions, including a change in the section title to "Removing genes that are lowly expressed", and we have added comments to point out that our analysis assumes no differential isoform usage. We have also simplified our example in the "Organising gene annotations" section.

Thanks for pointing out the inconsistencies in the figure captions. They have now been addressed. 
Indeed, it would be nice to make the colours consistent between the interactive and static MDS plots. We like the colours used in the static plot and can have the interactive version of the plot updated once color specification becomes available to the MDS plotting function in Glimma.

\section{Competing Interests: None}

Reviewer Report 23 June 2016

https://doi.org/10.5256/f1000research.9688.r14436

(c) 2016 MacDonald J. This is an open access peer review report distributed under the terms of the Creative Commons Attribution License, which permits unrestricted use, distribution, and reproduction in any medium, provided the original work is properly cited.

\section{James W. MacDonald}

1 Department of Environmental and Occupational Health Sciences, University of Washington, Seattle, WA, USA

2 Department of Environmental and Occupational Health Sciences, University of Washington, Seattle, WA, USA

In this manuscript the authors perform a step-by-step analysis of a public RNA-Seq data set, using the Bioconductor packages edgeR, limma, and Glimma. The analysis proceeds through four stages (data preparation, preprocessing, univariate analyses, and gene set testing), with each stage broken up into several discrete steps. Each step is clearly explained and is accompanied by R code and output, so the reader can follow along if desired.

The target audience for this paper appears to be someone with passing familiarity with both $\mathrm{R}$ and statistics, without requiring expertise in either. Most of the code should be easy to understand, and any complex sections are clearly explained in the text. Similarly, the authors outline the analytic choices they make (e.g., filtering out unexpressed genes, specifying model coefficients, etc) at a more accessible level.

While both edgeR and limma are well known, popular Bioconductor packages, Glimma is a new package that was released in April 2016. This package uses the d3.js JavaScript library to generate interactive HTML documents that can be viewed locally, rather than needing to be accessed from a server running $\mathrm{R}$ (as, say a shiny app requires). This is an exciting development, and is unfortunately not as compelling as it could be, if the Glimma plots were part of the HTML version of the manuscript rather than provided as links.

This is a well written paper, and is a useful contribution to the literature; while each package has either an extensive user's guide or vignette, by necessity these documents pertain only to the package at hand. Most RNA-Seq analyses require a combination of multiple packages to complete, and this paper provides a clear example. 


\section{Major comments}

None.

\section{Minor comments}

In the section 'Organising gene annotations', the code used to subset the one-to-many mappings is needlessly complex. Simply doing something like

genes <- genes[!duplicated(genes[,1]),]

will accomplish the same thing, in a more straightforward way.

In the section 'Removing heteroscedasticity from count data', the authors state:

"When operating on a DGEList-object, voom converts raw counts to log-CPM values by automatically extracting library sizes and normalisation factors stored in the object. For a matrix of counts, the method of normalisation can be specified within voom using the normalize.method (by default no normalisation is performed)."

This is confusing; the authors already showed in an earlier section ('Normalising gene expression distributions') that converting to log-CPM using TMM normalization factors will do a shiftnormalization (which is what voom will do in this instance). The normalize.method argument to voom specifies additional normalization methods that can be applied to the matrix of TMM normalized log-CPM values.

Competing Interests: No competing interests were disclosed.

\section{I confirm that I have read this submission and believe that I have an appropriate level of expertise to confirm that it is of an acceptable scientific standard.}

\section{Author Response 30 Nov 2016}

\section{Charity Law}

Dear James, thank-you for your comments. We have considered your suggestions and made changes to simplify the section on 'Organising gene annotations' and to clarify that voom offers additional normalisation ontop of the TMM-normsalisation that is carried out.

Competing Interests: None

\section{Comments on this article}




\section{Version 2}

Reader Comment 15 Feb 2017

\section{Marc Teunis}

Dear authors,

I am testing the workflow on a CentOS RStudio Server (see session info below). I just wanted to point out a minor glitch in the code for downloading the files for this workflow.

I have installed the workflow from BIOCONDUCTOR using:

source("http://bioconductor.org/workflows.R")

workflowInstall("RNAseq123")

The current code chunck for downloading the file should be changed to

utils::download.file(url, destfile="GSE63310_RAW.tar", mode="wb", method = "wget")

Not setting method to "wget" results in an incomplete download of the RAW.tar archive.

Hoping to have aided future users with this comment.

Sincerely,

Marc

output sessionInfo() (partly)

R version 3.3.2 (2016-10-31)

Platform: x86_64-redhat-linux-gnu (64-bit)

Running under: CentOS Linux 7 (Core)

locale:

[1] LC_CTYPE=en_US.UTF-8 LC_NUMERIC $=$ C

[3] LC_TIME=en_US.UTF-8 LC_COLLATE=en_US.UTF-8

[5] LC_MONETARY=en_US.UTF-8 LC_MESSAGES=en_US.UTF-8

[7] LC_PAPER=en_US.UTF-8 LC_NAME $=C$

[9] LC_ADDRESS $=C$ LC_TELEPHONE $=C$

[11] LC_MEASUREMENT=en_US.UTF-8 LC_IDENTIFICATION $=C$

$\cdots$

Competing Interests: No competing interests were disclosed. 
The benefits of publishing with F1000Research:

- Your article is published within days, with no editorial bias

- You can publish traditional articles, null/negative results, case reports, data notes and more

- The peer review process is transparent and collaborative

- Your article is indexed in PubMed after passing peer review

- Dedicated customer support at every stage

For pre-submission enquiries, contact research@f1000.com 